\title{
Identification of Potential Biomarkers for Anti-PD-1 Therapy in Melanoma by Weighted Correlation Network Analysis
}

\author{
Xuanyi Wang ${ }^{1,+}$, Zixuan Chai $^{1,+}{ }^{+}$, Yinghong Li $^{2}{ }^{\mathbb{D}}$, Fei Long ${ }^{1}{ }^{\mathbb{C}}$, Youjin Hao ${ }^{3}$, Guizhi Pan ${ }^{1}$, \\ Mingwei Liu ${ }^{1, *(\mathbb{D})}$ and $\mathrm{Bo} \mathrm{Li}^{3, *(\mathbb{D})}$ \\ 1 Key Laboratory of Clinical Laboratory Diagnostics, College of Laboratory Medicine, Chongqing Medical \\ University, Chongqing 400046, China; xuanyi_w@163.com (X.W.); bioczx@stu.cqmu.edu.cn (Z.C.); \\ leonardfei@163.com (F.L.); panpan.helen@gmail.com (G.P.) \\ 2 School of Biological Information, Chongqing University of Posts and Telecommunications, \\ Chongqing 400065, China; liyinghong@cqupt.edu.cn \\ 3 College of Life Sciences, Chongqing Normal University, Chongqing 401331, China; haoyoujin@hotmail.com \\ * Correspondence: liumingwei@cqmu.edu.cn (M.L.); libcell@cqnu.edu.cn (B.L.) \\ + These authors contributed equally to this work.
}

Received: 2 March 2020; Accepted: 14 April 2020; Published: 17 April 2020

check for updates

\begin{abstract}
Melanoma is the most malignant form of skin cancer, which seriously threatens human life and health. Anti-PD-1 immunotherapy has shown clinical benefits in improving patients' overall survival, but some melanoma patients failed to respond. Effective therapeutic biomarkers are vital to evaluate and optimize benefits from anti-PD-1 treatment. Although the establishment of immunotherapy biomarkers is well underway, studies that identify predictors by gene network-based approaches are lacking. Here, we retrieved the existing datasets (GSE91061, GSE78220 and GSE93157, 79 samples in total) on anti-PD-1 therapy to explore potential therapeutic biomarkers in melanoma using weighted correlation network analysis (WGCNA), function validation and clinical corroboration. As a result, 13 hub genes as critical nodes were traced from the key module associated with clinical features. After receiver operating characteristic (ROC) curve validation by an independent dataset (GSE78220), six hub genes with diagnostic significance were further recovered. Moreover, these six genes were revealed to be closely associated not only with the immune system regulation, immune infiltration, and validated immunotherapy biomarkers, but also with excellent prognostic value and significant expression level in melanoma. The random forest prediction model constructed using these six genes presented a great diagnostic ability for anti-PD-1 immunotherapy response. Taken together, IRF1, JAK2, CD8A, IRF8, STAT5B, and SELL may serve as predictive therapeutic biomarkers for melanoma and could facilitate future anti-PD-1 therapy.
\end{abstract}

Keywords: melanoma; anti-PD-1 therapy; WGCNA; biomarker

\section{Introduction}

Melanoma, as a form of highly aggressive skin cancer, is easy to metastasize and thus difficult to treat [1]. Recent clinical studies with anti-PD-1 immunotherapy have shown superior clinical efficacy and significant survival benefits for melanoma patients [2]. However, only a portion of the patients have an objective response to PD-1 blocking immunotherapy [3], and the remaining ones show little or no response, even involving in a high-grade immune-related adverse event $[2,4,5]$. Moreover, the use of immunotherapy would greatly increase the medical cost for an individual patient [6,7]. Regarding the clinical response, costs and side effects, it is urgent that predictive 
biomarkers are needed for assistance in identifying which patients are more prone to benefit from checkpoint inhibitor-based immunotherapy.

To date, it has been noticed that the clinical efficacy of anti-PD-1 therapy is relevant to the intrinsic features of tumor cells and tumor microenvironment (TME) or gene signatures. Some characteristics such as tumor mutational burden, deficiency of mismatch repair, host expression of PD-1/PD-L1 and the density of tumor-infiltrating lymphocytes have been reported as promising biomarkers for anti-PD-1 therapy [8]. Of which, the expression of PD-L1 typifies the most well-focused potential biomarker for the anti-PD-1 immune checkpoint blockade therapy response [9]. Several studies have revealed that PD-L1 expression played a crucial role in enriching the anti-tumor response for pre-treatment melanoma specimens, and 30-40\% of melanoma patients with high expression levels of PD-L1 had durable and objective responses [10]. The clinical research by Cottrell, T.R. et al. addressed a similar result [11].

However, PD-L1 expression is dynamic and varies over time, which leads to poor anti-PD-1 efficacy [8]. For instance, Patel, S.P. et al. proposed that during melanoma, lung, kidney, and other cancers, over $36 \%$ of patients with positive PD-L1 IHC expression responded to the PD-1/L1 axis-directed therapy, but approximately $17 \%$ of patients with a negative expression were also responded [12]. Johnson, D.B. et al. found that PD-L1 expression had no predictive power for survival, but the interaction of PD-1/PD-L1 and IDO-1/HLA-DR co-expression may improve outcomes of anti-PD-1 therapy in melanoma [13].

Previous studies indicated that exploring tumor type-specific gene expression, dynamic omics profiles in intratumoral heterogeneity, the tumor cell infiltrate, and the tumor-host microenvironment was probably helpful for the establishment of anti-PD-1 therapeutic biomarkers [14-16]. For example, with genomic arrays from 14,492 distinct solid tumors, Messina, J.L. et al. discovered a novel expression signature of 12 chemokine genes that lead to a potentially suitable selection for improving immunotherapy in melanoma [17]. Ayers, M. et al. quantified T cell-inflamed gene-expression profiles in the microenvironment, and it was developed for pembrolizumab trials in melanoma [18]. Ribas, A. et al. confirmed that the expression signatures of interferon-inflammatory immune genes were related to the overall response rate in patients treated with pembrolizumab [19]. By evaluating the gene signatures of six immune cells, Varn, F.S. et al. found that the B cell-derived expression signature could predict the checkpoint inhibitor-based immunotherapy response for patients [20].

Although several studies have a start on biomarkers for anti-PD-1 benefits in melanoma, few of them focused on the functional correlation between the genes as well as the relationship between gene expressions and therapeutic response. The above studies limited the development and exploration of biomarkers and the molecular mechanisms to a systems biology perspective [21,22]. Therefore, a robust analysis based on omics data and gene co-expression network was applied in this study to provide an insight into the correlation among genes as well as between the genes and therapeutic response, which lead to the identification of potential therapeutic biomarkers of anti-PD-1 immunotherapy in melanoma.

In this study, all the existing RNA-seq datasets on anti-PD-1 immunotherapy of melanoma were reanalyzed using a bioinformatics approach, including GSE91061, GSE78220 and GSE93157 (79 samples in total). With the weighted correlation network analysis, the dataset of GSE91061 was adopted to construct gene co-expression network across the different samples, followed by the identification of key modules with therapeutic response. Functional enrichment analysis was further used to investigate the biological function of the key module genes. From this, the hub genes were identified based on the co-expression network, protein-protein interaction (PPI) network and gene topological network. GSE78220, as an independent dataset, was utilized to investigate the potential diagnostic genes for anti-PD-1 therapy from hub genes, which were plotted by receiver operating characteristic (ROC) analysis. Afterward, function enrichment, immune infiltration level, gene expression level, overall survival, and gene correlation of potential diagnostic genes were validated by Gene Set Variation Analysis (GSVA), Tumor Immune Estimation Resource (TIMER), and Gene Expression Profiling 
Interactive Analysis (GEPIA). A prediction model of immunotherapy was constructed by the hub genes based on random forest classifier. The accuracy of the prediction model was verified based on GSE93157.

\section{Materials and Methods}

The workflow used in the study is shown in Figure 1, which included three main steps, hub gene identification, diagnostic efficiency analysis and validation of potential therapeutic biomarkers.

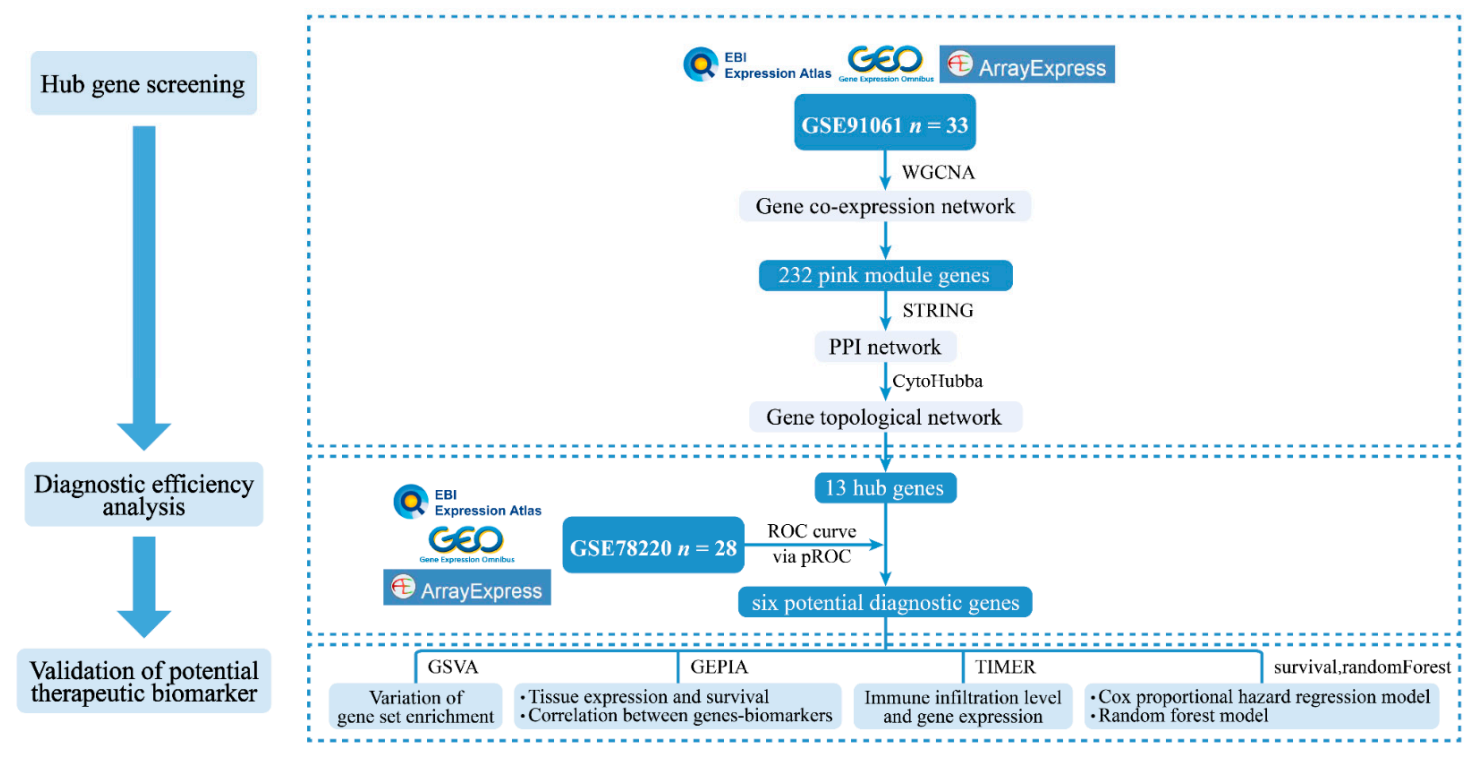

Figure 1. The flowchart of study design. Flow diagram of analysis procedure including discovery, analysis and validation of potential therapeutic biomarkers in this study. GEO: Gene Expression Omnibus; WGCNA: Weighted correlation network analysis; PPI: Protein-protein interaction; ROC: Receiver operating characteristic; GSVA: Gene set variation analysis; GEPIA: Gene expression profiling interactive analysis; TIMER: Tumor immune estimation resource.

\subsection{Data Acquisition of Gene Expression Datasets}

Using the keywords such as "PD-1", "immunotherapy", "therapy", "treatment", and "melanoma", datasets were investigated from the main transcriptomics database, including Gene Expression Omnibus (GEO) (http://www.ncbi.nlm.nih.gov/geo) [23], ArrayExpress (https://www.ebi.ac.uk/arrayexpress) [24] and Expression Atlas (https://www.ebi.ac.uk/gxa/home) [25]. By manually checking, the raw datasets meeting the following standards were retained in subsequent analyses: (i) inclusion of gene expression data of responders (complete response or particle response) or non-responders (progress disease) to anti-PD-1 therapy in melanoma. The responders and non-responders to immunotherapy were defined according to iRECIST guidelines [26], and the stable disease was not included due to the controversial role in response to therapy [27,28]; (ii) at least 15 accessible samples in the datasets; and (iii) availability of raw sequence or microarray data.

\subsection{Construction of Gene Co-Expression Network}

The Dataset GSE91061 [27] (10 complete response or particle response samples and 23 non-response samples) was normalized with DESeq2 [29], and the genes were ranked by median absolute deviation (MAD). The top 5000 genes with the highest MADs were extracted for gene co-expression network construction via the weighted correlation network analysis (WGCNA) package [30]. Pearson's correlation coefficients were calculated between each pair of the extracted genes to generate the adjacency matrix. Then, the function "tomlikeity" was used to transform the adjacency matrix into the topological overlap measure (TOM). The TOM reflected the correlative interconnectivity between two 
genes according to their degree of shared adjacency for the whole network [30]. The genes with similar expression patterns were clustered into the same module utilizing the average linkage hierarchical clustering based on the TOM-based dissimilarity measure.

\subsection{Identification of Clinically Significant Modules}

Two approaches were utilized to obtain the modules significantly associated with clinical traits. Pearson's correlation analysis was adopted to calculate the correlation between clinical features and the module eigengenes (MEs). MEs were the major component for each gene module and the most representative expression patterns of the module [30]. Then, the gene significance (GS) and module significance (MS) were calculated. GS represented the correlation between gene expression and clinical features. MS was the average GS across all genes in the module [30]. Generally, the module with the first-ranked MS was considered as the clinically significant modules.

\subsection{Gene Ontology (GO) and Kyoto Encyclopedia of Genes and Genomes (KEGG) Pathway Enrichment Analysis}

To investigate the function of the genes in clinically significant modules, enrichment analysis of Gene Ontology (GO) was performed by NetworkAnalyst (https://www.networkanalyst.ca) [31]. GO terms were regarded as the significant ones when the adjusted $p$-value $<5 \times 10^{-3}$ and minimum gene counts $>20$. NetworkAnalyst was also applied for performing Kyoto Encyclopedia of Genes and Genomes (KEGG) pathway enrichment analysis. Adjusted $p$-value $<2 \times 10^{-4}$ and the minimum gene counts $>10$ were regarded as the cut-off criteria.

\subsection{Identification of Hub Genes Based on Protein-Protein Interaction (PPI) and Topological Network}

The PPI network of key module genes was constructed by the STRING database (http://string-db. org) [32]. The plug-in CytoHubba [33] of Cytoscape software [34] was applied to explore key nodes in the PPI network via 11 topological algorithms including Degree, Edge Percolated Component, Maximum Neighborhood Component, Density of Maximum Neighborhood Component, Maximal Clique Centrality, Bottleneck, EcCentricity, Closeness, Radiality, Stress, and Betweenness [33]. The top 50 nodes were defined as core genes for each algorithm in the topological network. The intersected core genes derived from the 11 topological algorithms were considered as the hub genes with important biological regulatory functions.

\subsection{Receiver Operating Characteristic (ROC) Curve Analysis}

To verify the diagnostic ability of hub genes in another dataset, the RNA-sequencing dataset GSE78220 [28] was utilized, which included 28 melanoma patient samples (five complete response samples, 10 partial response samples and 13 non-response samples) treated with anti-PD-1 therapy. Using pROC package [35], the area under the ROC curve (AUC) was calculated on the expression data of each hub gene. In addition, to obtain test results with high specificity, we focused on the partial area under the curve (pAUC) between 90\% and 100\% specificity [36], which was also calculated by the pROC package [35]. In the present study, the larger AUC or pAUC value for a gene indicated that this gene can better distinguish responders from non-responders for anti-PD-1 immunotherapy [37]. Based on the AUC or pAUC values, the diagnosis effect of hub genes was further investigated.

\subsection{Gene Set Variation Analysis of Hub Genes}

GSVA is a reliable approach to evaluate the variation of function activity across different samples via an unsupervised manner [38]. To explore the functions most associated with hub genes, "GSVA" R package was applied based on the GSE78220 [28]. The gene set "c5.all.v2.5.symbols.gmt" was downloaded from the Molecular Signature Database (MSigDB) accessed on 5 December 2019 (http://software.broadinstitute.org/gsea/msigdb/index.jsp) as the reference gene set, and $p$-value $<0.05$ was used as the cut-off point. 


\subsection{Correlation Analysis of Hub Genes and Immune Infiltration Level}

The online tool TIMER (https://cistrome.shinyapps.io/timer) database [39] was used to explore the association between the hub gene expression and immune infiltration levels in melanoma. Based on deconvolution of the previously published statistical methods [40], the TIMER database is a comprehensive resource to evaluate the abundance of tumor-infiltrating immune cells (TIICs) across diverse cancer types from The Cancer Genome Atlas (TCGA) database (https://cancergenome.nih.gov).

\subsection{Validation and Survival Analysis of Hub Genes}

GEPIA database (http://gepia.cancer-pku.cn/index.html) provides comprehensive expression analyses for RNA sequencing data, including 9,736 tumors and 8,587 normal samples from TCGA and the GTEx projects [41]. To reveal the expression level and prognostic value of hub genes in melanoma, differential expression analysis ( $p$-value $<0.05$ and $\left.\log _{2} \mathrm{FC}>1\right)$ and survival analysis $(p$-value $<0.05)$ were performed via GEPIA. In addition, the Cox proportional hazard regression model of the screened hub genes was constructed via the survival package in $\mathrm{R}$ software to evaluate the overall survival of the melanoma patients. The Cox proportional hazard regression model is a very useful tool to access the impact of lifetime-related factors on the hazard function [42]. The skin cutaneous melanoma (SKCM) and uveal tract melanoma (UVM) datasets from TCGA as well as normal samples from GTEx projects were used for analysis.

\subsection{Correlation Analysis of Hub Genes and Biomarkers of Anti-PD-1 Therapy}

GEPIA was also utilized to identify the relationship of hub genes and biomarkers of anti-PD-1 immunotherapy. The SKCM and UVM datasets from TCGA were used for analysis. The Spearman method was adopted to calculate the correlation coefficient between genes. The terms with $p$-value $<0.05$ were regarded as statistically significant.

\subsection{Random Forest}

A prediction model of anti-PD-1 immunotherapy response was constructed via the random forest classifier. The hub genes were the covariates of the prediction model. The random forest is a popular tool for classification and regression, which shows a powerful ability to construct a predictive model for new biomarkers. The random forest is less prone to over-fitting problems and can handle a large amount of noise. A random forest-based classifier was built via the randomForest package in $\mathrm{R}$ software based on the algorithm of Breiman and Cutler [43]. The samples of GSE78220 $(n=28)$ [28] were randomly divided into the training set and test set via the caret package, each of which contained 14 samples. Then, the decision tree model of the training set was established to obtain the classification. Next, the classification results of each time were averaged to calculate the final classification. The model built by the training set would be tested by the test set. Each result would calculate the error rate through Out-of-bag (OOB) to evaluate the correct rate of the combined classification. OOB was the data not sampled when the training set was randomly sampled. The OOB samples were used to estimate the prediction error and variable importance [44]. Finally, the melanoma samples treated with anti-PD-1 therapy of GSE93157 [45] (seven complete response or particle response samples and 11 non-response samples) were used as the validation set to verify the accuracy of the random forest model. AUC index was utilized to evaluate the efficiency of the prediction model.

\section{Result}

\subsection{Construction of Weighted Co-Expression Network and Identification of Key Modules}

According to the strict standards described above, GSE91061 $(n=33)$ [27], GSE78220 $(n=28)$ [28] and GSE93157 $(n=18)$ [45] were retained for further analysis. To determine the key modules connected with clinical features (therapeutic response), the weighted co-expression network was constructed by 
WGCNA based on the GSE91061 $(n=33)$. The power of $\beta=5$ (scale free $R^{2}=0.89$ ) was selected as the soft-thresholding parameter to ensure a scale-free network (Figure 2A). A total of 27 modules, ranging in size from 30 to 525 genes, was found by the average linkage hierarchical clustering (Figure 2B-C). Based on the calculation of Pearson's correlation coefficient, the pink module was considered as the highest correlation one with the clinical traits ( $\operatorname{cor}=0.38, p$-value $=0.03$ ) (Figure 2D). Moreover, the pink module showed the highest MS in Figure 2E. Modules with a greater MS were relevant to the clinical traits. According to the results of the two methods, the pink module was selected as a key module to be studied in subsequent analyses.

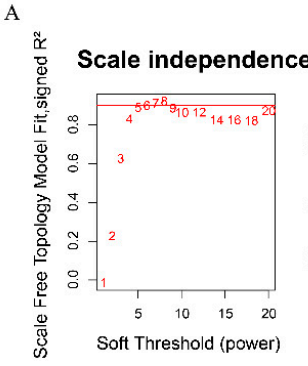

C

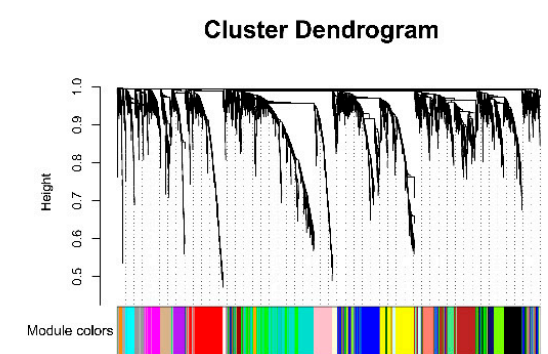

E
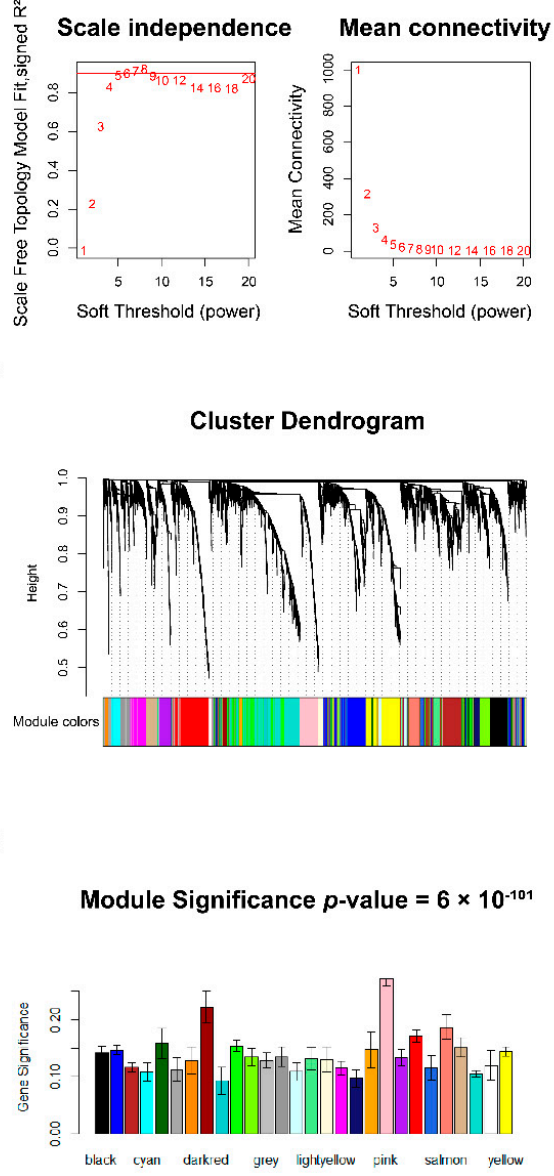

$\mathrm{B}$

$\mathrm{D}$

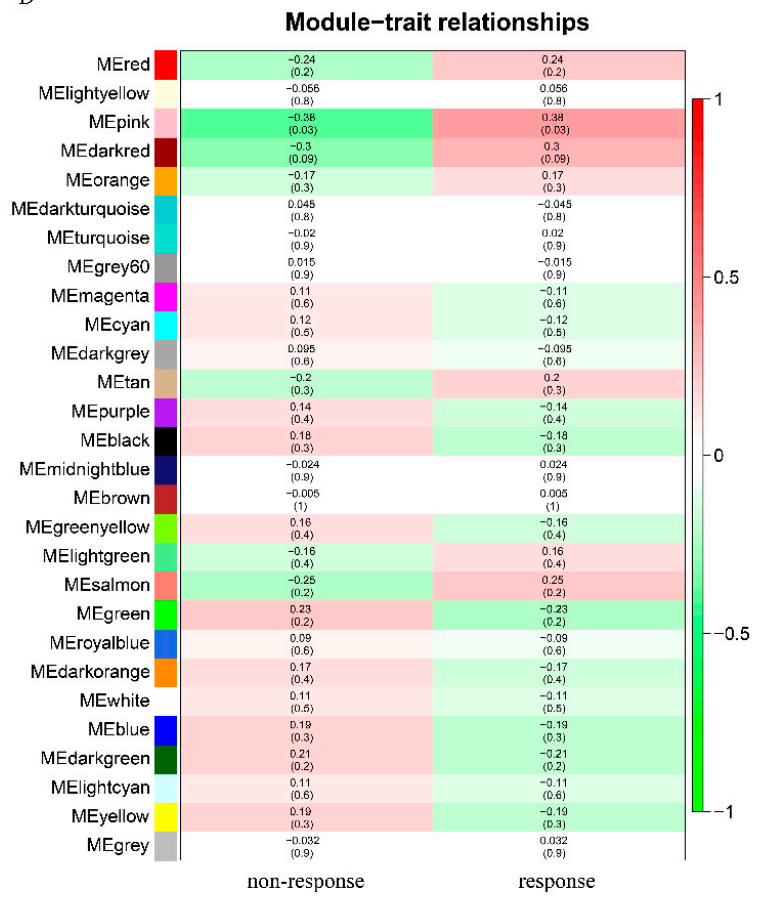

Figure 2. Identification of key modules connected with clinical features through WGCNA. (A) The left and right panel showed the scale-free fit index and the mean connectivity for various soft-thresholding powers, respectively. When the soft-thresholding powers ( $\beta$ ) equaled five, the average degree of connectivity was close to zero. (B) The cluster dendrogram of module eigengenes. (C) The cluster dendrogram of 5,000 module eigengenes from the GSE91061 dataset. Each branch in the figure represented one gene, and every color below represented one co-expression module. (D) Heatmap of the correlation between module eigengenes and clinical traits of anti-PD-1 immunotherapy responsiveness. The color of cells in the heatmap represented the correlation coefficients of different sizes. Specifically, red colors represented the positive correlations and green colors stood for the negative correlations. The figure without brackets in each cell indicated the clinical feature correlation coefficients. The corresponding $p$-value was shown below in parentheses. The pink module was significantly correlated with response to anti-PD-1 therapy. (E) Distribution of average gene significance and errors in the modules associated with the response to anti-PD-1 therapy. 


\subsection{Potential Functional Roles of Genes in the Pink Module}

The results showed $19 \mathrm{GO}$ terms were enriched with adjusted $p$-value $<5 \times 10^{-3}$ and minimum gene counts $>20$ (Figure 3A). The pink module genes were mainly enriched in immune function (e.g., immune response, immune progress, and immune cell activation), hemopoiesis and signal transduction. Activating the immune system via blocking the immune checkpoint was a crucial factor for anti-PD-1 immunotherapy to attack the tumor cells [46], which indicated that genes involved the immune function may be relevant to the efficacy of anti-PD-1 immunotherapy.

Based on KEGG pathway mapping, the pink module genes were significantly involved in 12 pathways (adjusted $p$-value $<2 \times 10^{-4}$, minimum gene counts $>10$ ) such as Epstein-Barr virus infection, chemokine signaling pathway, natural killer cell-mediated cytotoxicity, Th1 and Th2 cell differentiation, Jak-STAT signaling pathway, and so on (Figure 3B). Natural killer cell-mediated cytotoxicity was the fourth most significant pathway (adjusted $p$-value $=4.33 \times 10^{-5}$ ). Ardolino, M. et al. found that the natural killer cell response elicited by the PD-1/PD-L1 blockade played vital roles in the therapeutic effect of immunotherapy [47]. The results also demonstrated that many genes were significantly involved in the chemokine signaling pathway. Chemokine gene expression signatures including CCL5, CXCL9, CXCL10, and CXCL11 were reported that could accurately predict anti-PD-1 immunotherapy response for patients with head and neck squamous cell carcinoma and gastric cancer [18]. Furthermore, Herbst, R.S. et al. found that the expression of CXCL9 had a significant, positive correlation with the therapeutic response in melanoma [48]. Comparing melanoma samples with normal controls, Boots, A.M. et al. indicated that PD-1 checkpoint blockades enhanced the inflammatory responses of Th1 and Th17 as well as inhibited Th2 responses [49]. Genes involved in Th1 and Th2 cell differentiation may indirectly reflect the response of blockades. Additionally, the Jak-STAT signaling pathway was also enriched. Lu, C. et al. demonstrated that Jak-STAT signaling inhibited cytotoxic T lymphocyte activation to weaken the effect of anti-PD-1 immunotherapy [50].

$\Lambda$

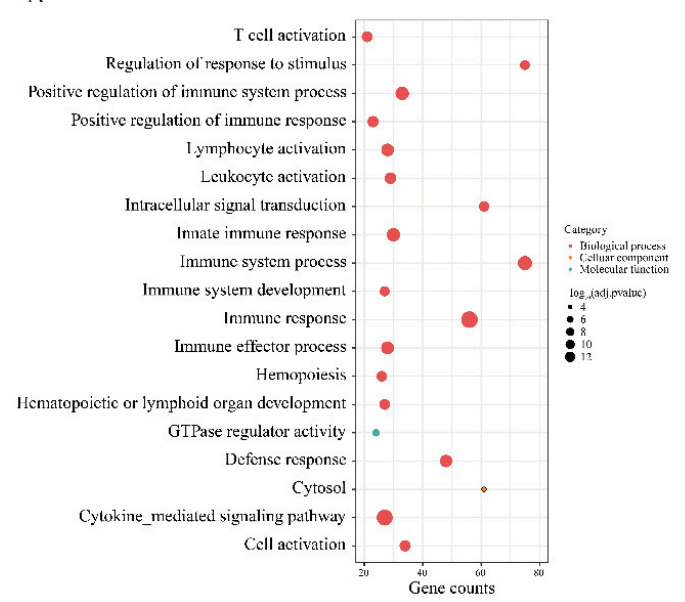

B

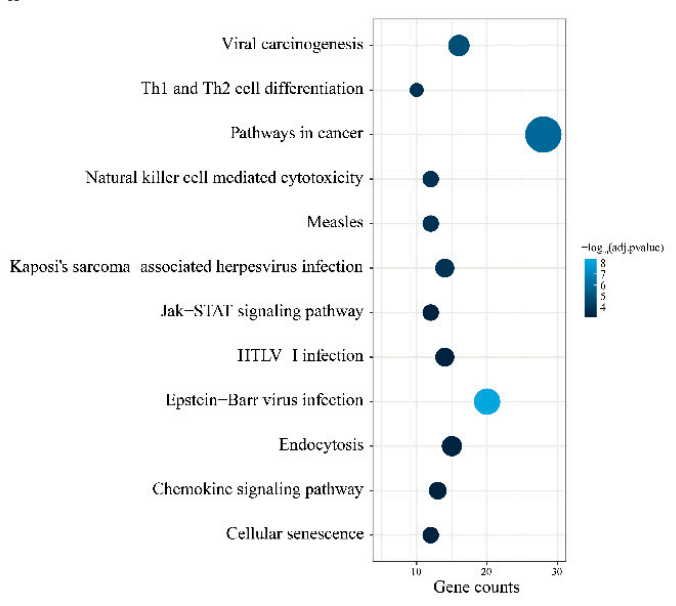

Figure 3. Enrichment analysis of gene ontology and Kyoto Encyclopedia of Genes and Genomes (KEGG) pathway for genes in the pink module. (A) Enrichment analysis of gene ontology. Annotation terms in the biological process, cellular component and molecular function were marked in red, yellow, and green, respectively. Bubble size represented the value of $-\log _{10}$-adjusted $p$-value of enrichment significance. (B) Enrichment analysis of KEGG pathway. Different colors represent the value of $-\log _{10}$-adjusted $p$-value of enrichment significance.

\subsection{Identification of $\mathrm{Hub}$ Genes}

A total of 232 genes in the pink module were analyzed by STRING database. A PPI network containing 100 nodes and 134 interactions was built with the medium confidence score $(>0.4)$. After importing the data into Cytoscape and running the CytoHubba program, the top 50 node genes were calculated as the core genes by 11 topological algorithms, respectively (Table S1). As a result, 
a total of 13 genes including BTK, CD3E, CD48, IL2RG, IL2RB, LCP1, TRIM21, IRF1, JAK2, CD8A, IRF8, $S T A T 5 B$, and SELL were calculated as the intersection of the core genes in 11 algorithms, which were considered to be hub genes.

\subsection{ROC Curve Analysis of Hub Genes}

To validate the predictive power of 13 hub genes for anti-PD-1 therapy in melanoma, ROC curve analysis was enabled utilizing GSE78220. Finally, the results suggested that the expression of six genes including IRF1, JAK2, CD8A, IRF8, STAT5B, and SELL had a significant ability to distinguish the responders from non-responders to anti-PD-1 therapy in melanoma with AUC $>0.6$ and pAUC $>0.7$ (Figure 4).
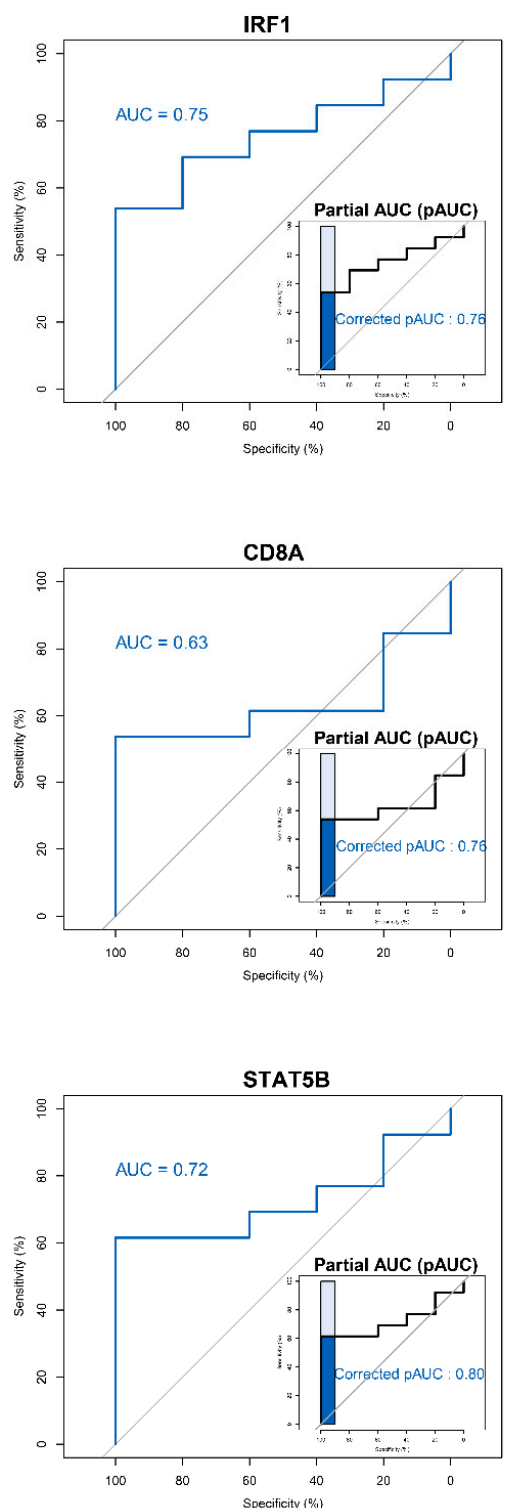

B

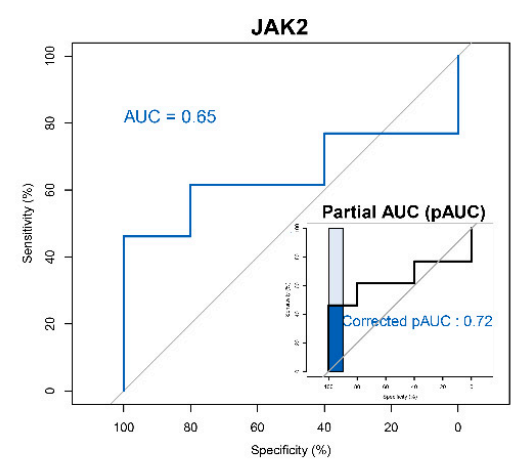

$\mathrm{D}$

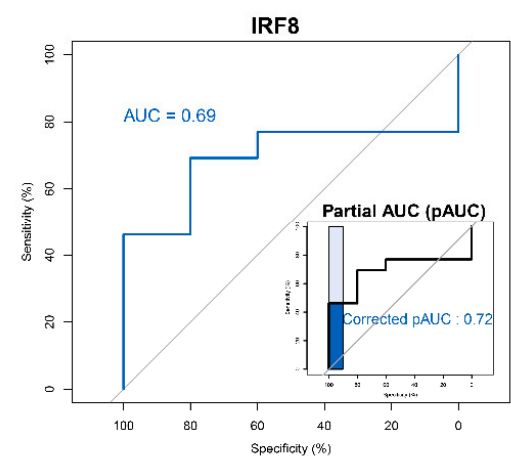

F

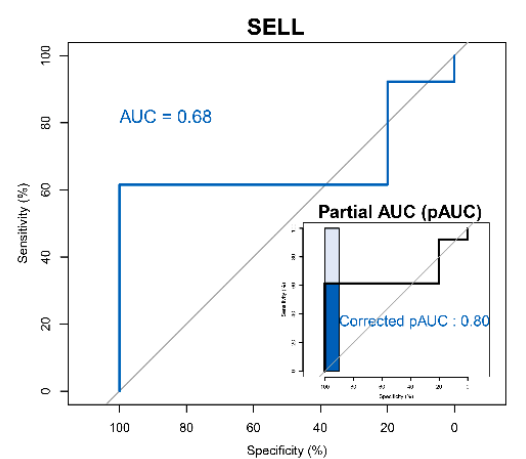

Figure 4. Receiver operating characteristic (ROC) curve analysis of six hub genes based on GSE78220. (A) IRF1, (B) JAK2, (C) CD8A, (D) IRF8, (E) STAT5B, and (F) SELL. The area under the ROC curve (AUC) and partial area under the curve (pAUC) are shown in each subgraph, and the pAUC is on the bottom right of the subgraph. The AUCs of IRF1, JAK2, CD8A, IRF8, STAT5B, and SELL were 0.75, 0.65, $0.63,0.69,0.72$, and 0.68 , respectively. The pAUCs of IRF1, JAK2, CD8A, IRF8, STAT5B, and SELL were $0.76,0.72,0.76,0.72,0.80$, and 0.80 , respectively. 


\subsection{Gene Expression of Hub Genes}

The expression of the six genes in responders or non-responders to anti-PD-1 therapy (GSE91061) was shown in Figure 5. The expressions of IRF1, JAK2, CD8A, IRF8, and SELL were down-regulated in the responders compared with non-responders ( $p$-value $<0.05$ and $\log _{2} \mathrm{FC}>0.5$ ). However, the expression of $S T A T 5 B$ had no significant changes between the responders and non-responders.

A

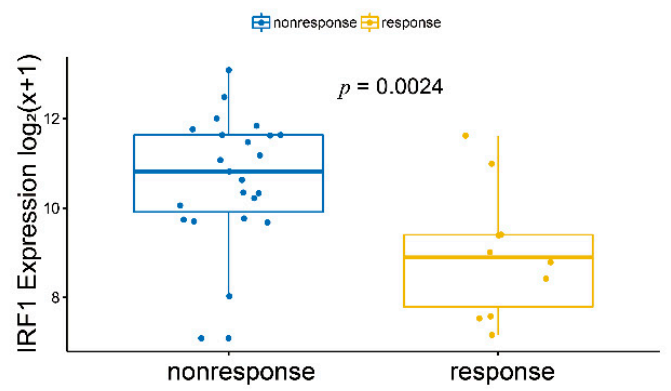

$\mathrm{C}$

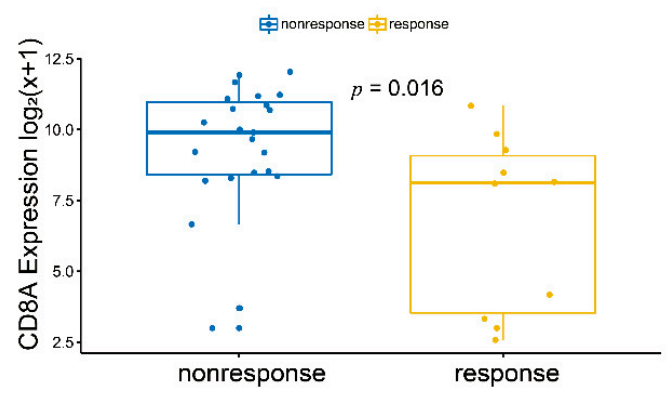

$\mathrm{E}$

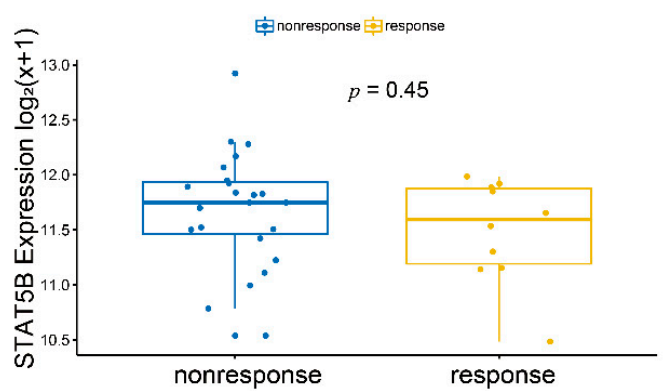

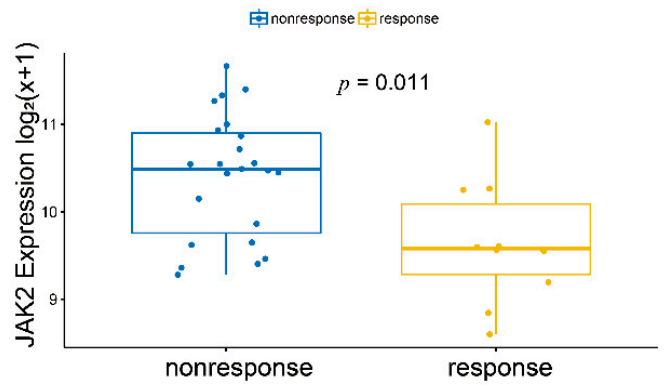

$\mathrm{D}$

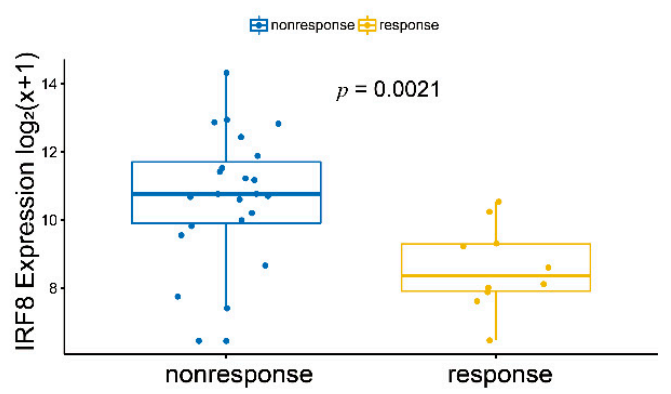

F

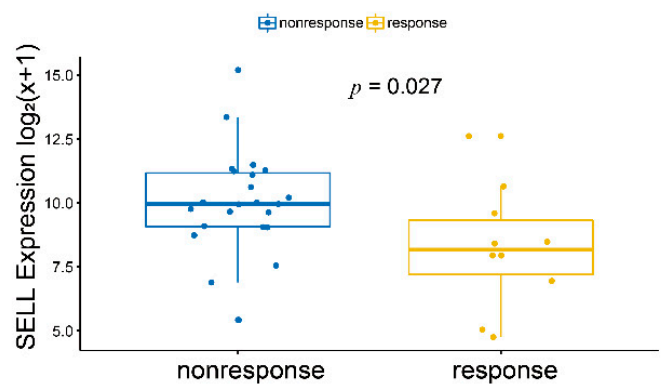

Figure 5. The expression of the six hub genes in responders or non-responders to anti-PD-1 therapy. (A) IRF1, (B) JAK2, (C) CD8A, (D) IRF8, (E) STAT5B, and (F) SELL gene expression differences between melanoma and normal tissues. The blue column represented the samples of non-responders, and the yellow column represented the samples of responders.

\subsection{Functional Enrichment Analysis by Gene Set Variation Analysis (GSVA)}

To further investigate the correlation between anti-PD-1 immune checkpoint blockade therapy and functional features of IRF1, JAK2, CD8A, IRF8, STAT5B, and SELL in melanoma, differential signature enrichment analysis was performed via GSVA based on the GSE78220 datasets $(n=28)$. A group of 27 functional signatures was enriched in RNA-seq data of 13 non-responding versus 15 responding pre-anti-PD-1 melanoma patients. The enrichment functions included regulation of transcription factor and promoter, lymphocyte proliferation and differentiation, regulation of hematopoiesis and the immune system, and so on (Figure 6). Most of the functions were up-regulated in the group with positive responses to immunotherapy and down-regulated in the group with negative responses to immunotherapy. This demonstrated that the functional signatures of $I R F 1, J A K 2, C D 8 A$, 
IRF8, STAT5B, and SELL could provide several suggestions for distinguishing the clinical effects of anti-PD-1 immunotherapy.

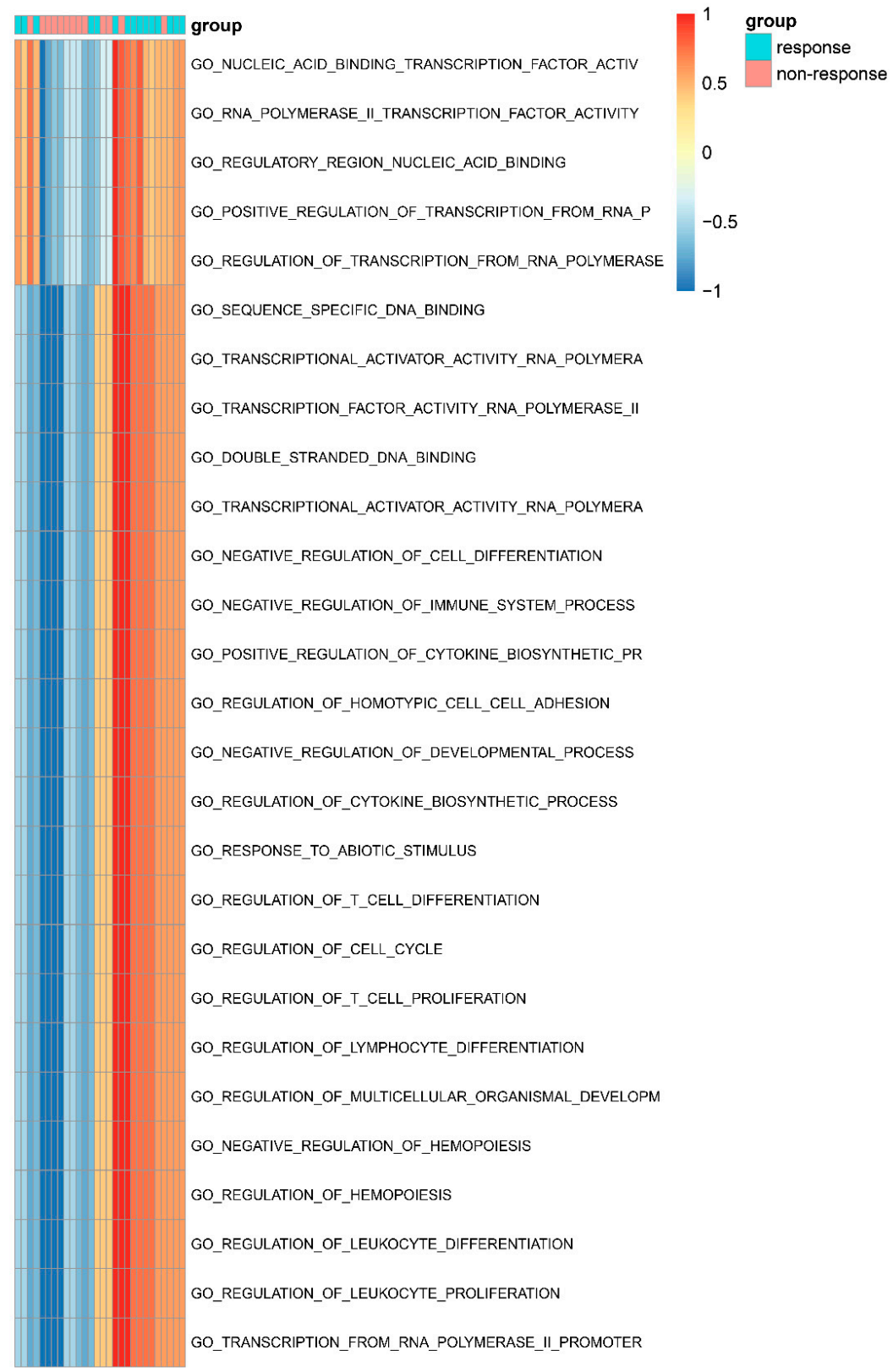

Figure 6. Gene set variation analysis of hub genes in the GSE78220 dataset. The functional analysis clustered gene ontology terms with $p$-value $<0.05$ for six hub genes. The $x$-axis represents melanoma samples treated with anti-PD-1 therapy, including responders (green) and non-responders (red). The $y$-axis shows the enriched GO terms, and the orange ones indicate the up-regulation of the function, while the blue ones indicate the downregulation.

\subsection{Analysis of Association Between Hub Genes and Immune Infiltration Level}

Recent studies confirmed that immune infiltrating lymphocytes, as a crucial factor in regulating the immune system, had vast potential to predict the effect of checkpoint inhibitor therapy [7]. In this study, the TIMER database was applied to analyze the relationship between the hub gene expressions and immune infiltration levels in melanoma. As a whole, the expressions of six hub genes in SKCM were associated with $\mathrm{B}$ cells, $\mathrm{CD} 4^{+} \mathrm{T}$ cells, $\mathrm{CD} 8^{+} \mathrm{T}$ cells, neutrophils, macrophages, and dendritic cells 
( $p$-value $<0.05)$ (Figure 7A-F). The overall trend of these six genes was negatively related to tumor purity, which demonstrated that these genes may be highly expressed in TME. The expressions of IRF1, CD8A and IRF8, especially IRF8, were significantly correlated with the infiltration of CD8 ${ }^{+} \mathrm{T}$ cells, neutrophils and dendritic cells (cor $>0.5, p$-value $<1 \times 10^{-5}$ ). Several studies revealed that $\mathrm{CD} 8^{+} \mathrm{T}$ cells, neutrophils and dendritic cells were related to better outcomes and longer survival for patients under immunotherapy [51-53], which implied IRF1, CD8A and IRF8 were probably connected with the prognostics of immune treatment. However, the correlation between the hub genes expression and immune infiltrating levels was not obvious in UVM.
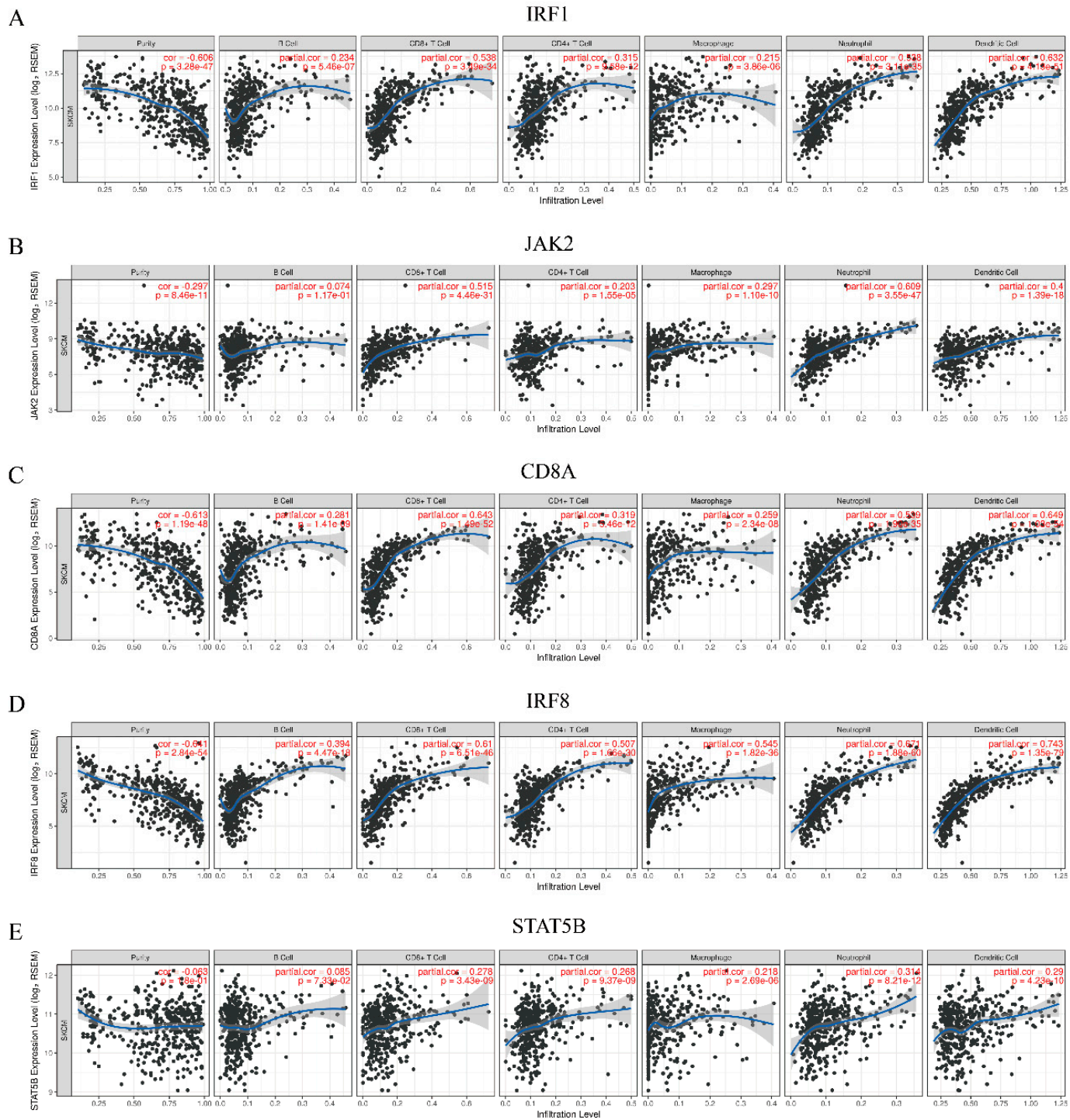

F

SELL

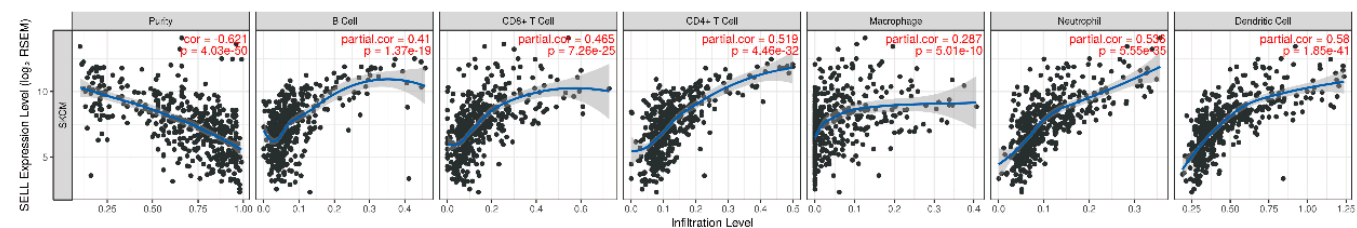

Figure 7. Correlation of six hub genes with immune infiltration in melanoma. (A) IRF1, (B) JAK2, (C) CD8A, (D) IRF8, (E) STAT5B, and (F) SELL. The x-axis demonstrates the immune infiltration levels. Spearman's correlation coefficient and $p$-value are shown in the upper right corner. 


\subsection{Validation of Hub Genes in The Cancer Genome Atlas (TCGA) Datasets}

The prognostic value and expression level of the six hub genes were validated by TCGA datasets on melanoma. The results indicated that $C D 8 A$ and SELL were up-regulated as well as JAK2 was down-regulated in melanoma samples compared with normal controls ( $p$-value $<0.05$ ) (Figure $8 \mathrm{~A}$ ). Furthermore, the results of the survival analysis suggested that high expressions of IRF1, JAK2, CD8A, IRF8, and SELL correlated significantly with improved clinical outcomes ( $p$-value $<0.01$ ) (Figure 8B). In addition, the multivariate Cox regression analyses for the six genes including IRF1, JAK2, CD8A, IRF8, STAT5B, and SELL were performed. The Cox proportional hazard regression model equation is as follows:

$$
\begin{gathered}
\text { Risk }_{a}=-0.127546 \cdot \text { IRF1 }-0.138979 \cdot J A K 2-0.098595 \cdot \text { CD } 8 A+0.132417 \cdot \text { IRF } 8- \\
0.070296 \cdot \text { STAT5B }-0.009357 \cdot \text { SELL }
\end{gathered}
$$

The risk score was calculated for each sample, and the samples were grouped according to the median risk score (cutoff $=-3.376457$ ). The results showed that the prognoses of the high-risk and low-risk groups significantly differed (Figure $8 C$ ). High expression of IRF8 was related to a high risk of death. The high expression of IRF1, JAK2, CD8A, STAT5B, and SELL was connected with a low risk of death and was a protective factor of melanoma. The results were roughly consistent with the survival analysis of a single gene. In previous studies, anti-PD-1 immunotherapy usually showed a remarkable ability to effectively improve the survival benefits of patients across several cancers [54]. Patients with favorable prognosis frequently showed a positive response to anti-PD-1 therapy [55]. Hence, combining with the results of survival and Cox regression analyses, it implied that the high expressions of IRF1, JAK2, CD8A, and SELL may predict a positive response to anti-PD-1 immunotherapy for melanoma patients.

a

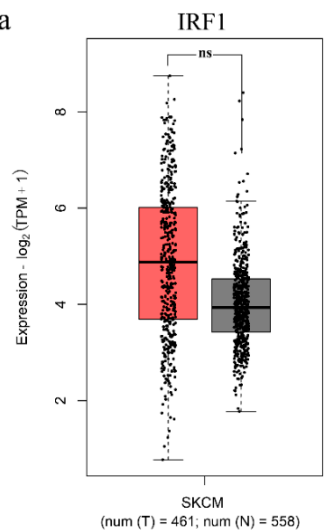

d

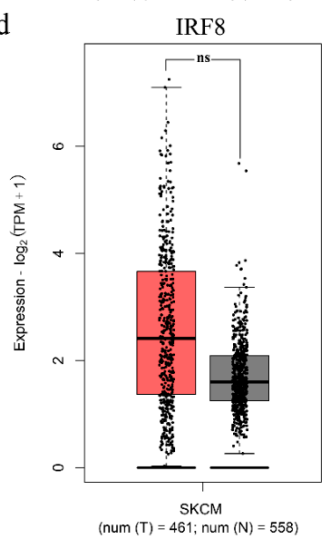

b

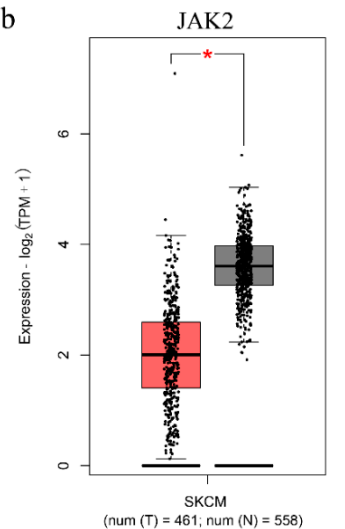

e

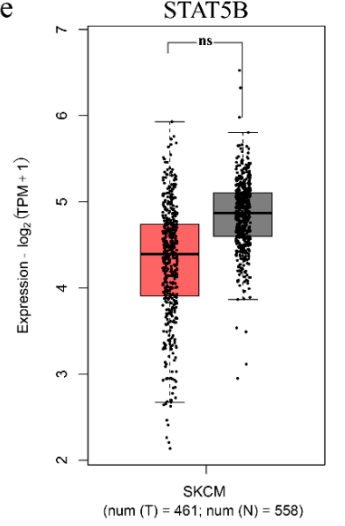

(A)
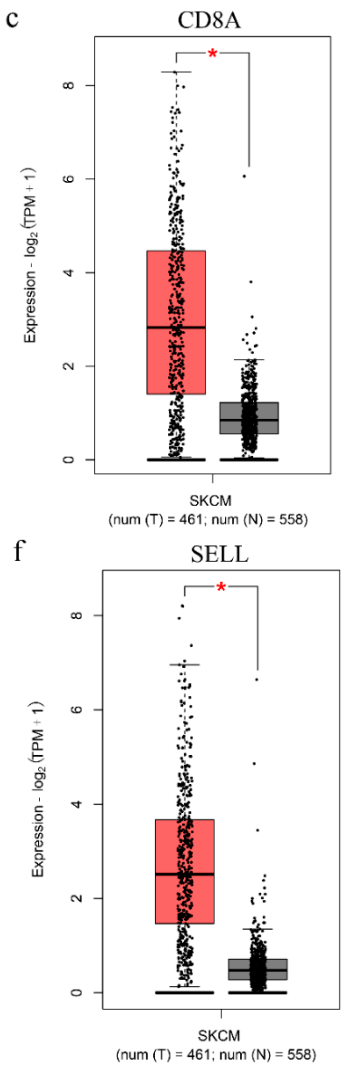

Figure 8. Cont. 
a

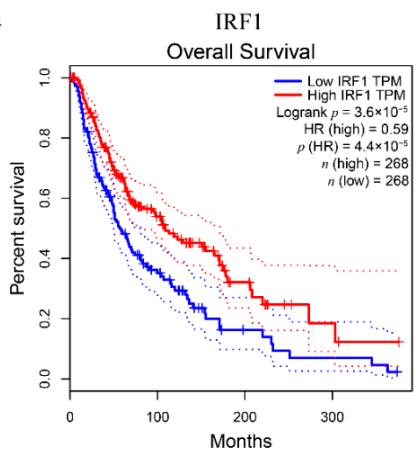

d

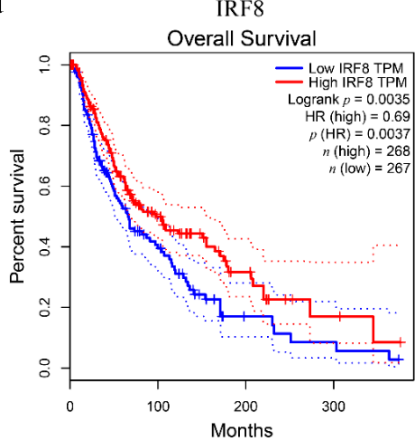

b

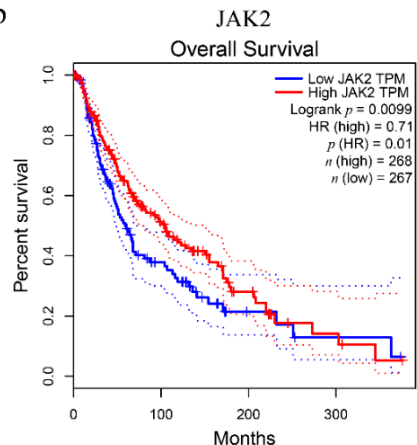

$\mathrm{e}$

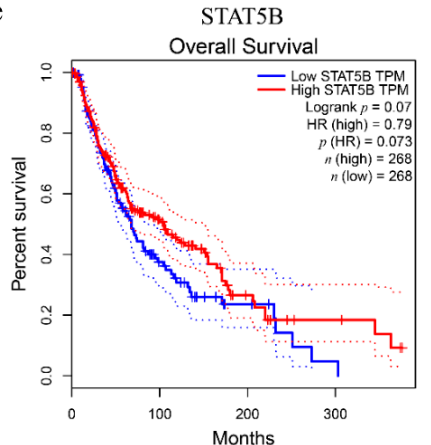

c

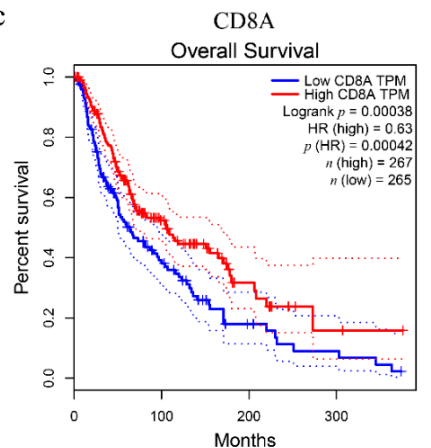

f

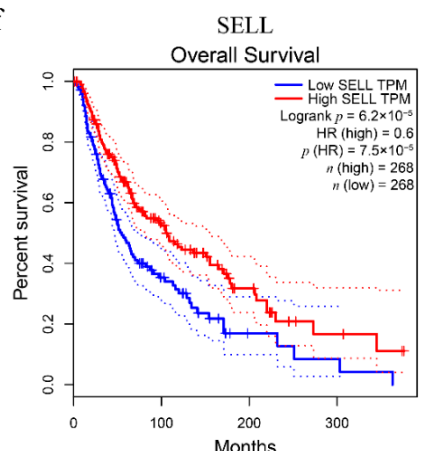

(B)

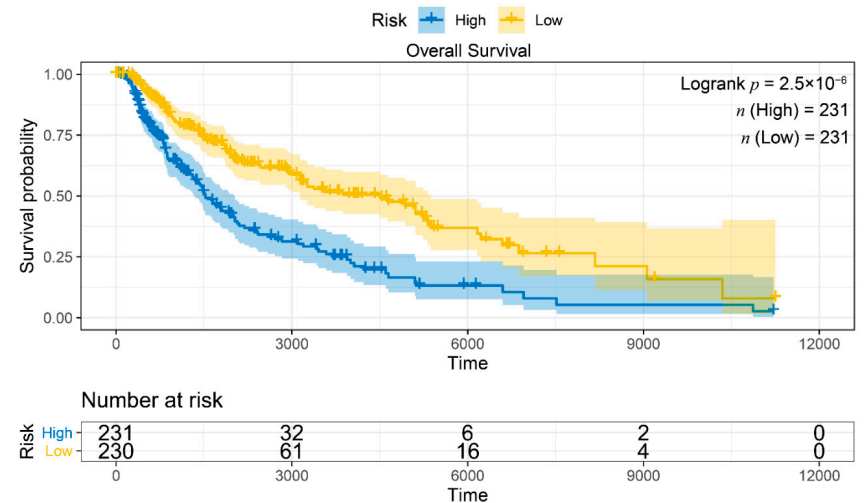

(C)

Figure 8. Validation of the expression level and prognostics in melanoma for six hub genes. (A) IRF1, JAK2, CD8A, IRF8, STAT5B, and SELL gene expression differences between melanoma and normal tissues. The red column represents the melanoma samples, and the black column represents the normal samples. (B) Survival analysis of IRF1, JAK2, CD8A, IRF8, STAT5B, and SELL in melanoma. The red line designates the samples with highly expressed genes, and the blue line indicates the samples with lowly expressed genes. (C) The multivariate Cox regression analysis of the six screened hub genes in overall survival. The horizontal axis (x-axis) represents time in days, and the vertical axis (y-axis) shows the probability of survival or the proportion of people surviving. The lines represented the survival curves of the two groups. ns, $p$-value $>0.05 ;{ }^{*}, p$-value $<0.05$.

\subsection{Relationships between Hub Genes and Biomarkers of Anti-PD-1 Therapy}

The relevance of gene expression between the six hub genes and the reported biomarkers (PD-L1/CD274, CXCR3 and IFN- $\gamma /$ IFNG) with significant predictive power for immunotherapy were analyzed by the GEPIA database. The expression level of PD-L1 IHC in tumor cells has been indicated as a unique biomarker of the immune checkpoint blockade response in the clinical [56]. CXCR3 and 
IFN- $\gamma$ have been confirmed by more than one article as biomarkers for sensitivity to the PD- 1 blockade based on clinical experiments and mouse models [18,57-59]. The results of correlation analysis showed a significant, positive correlation (cor $>0.6, p$-value $<0.05$ ) between the expression of four hub genes (IRF1, CD8A, IRF8, and SELL) and three biomarkers in melanoma (Figure 9A-C). Genes with strong correlation may have similar regulatory capacities or biological functions [60]. Thus, we speculated that the four genes closely related to PD-L1, IFN- $\gamma$ and CXCR3 may be connected with the efficacy evaluation of anti-PD-1 immunotherapy.
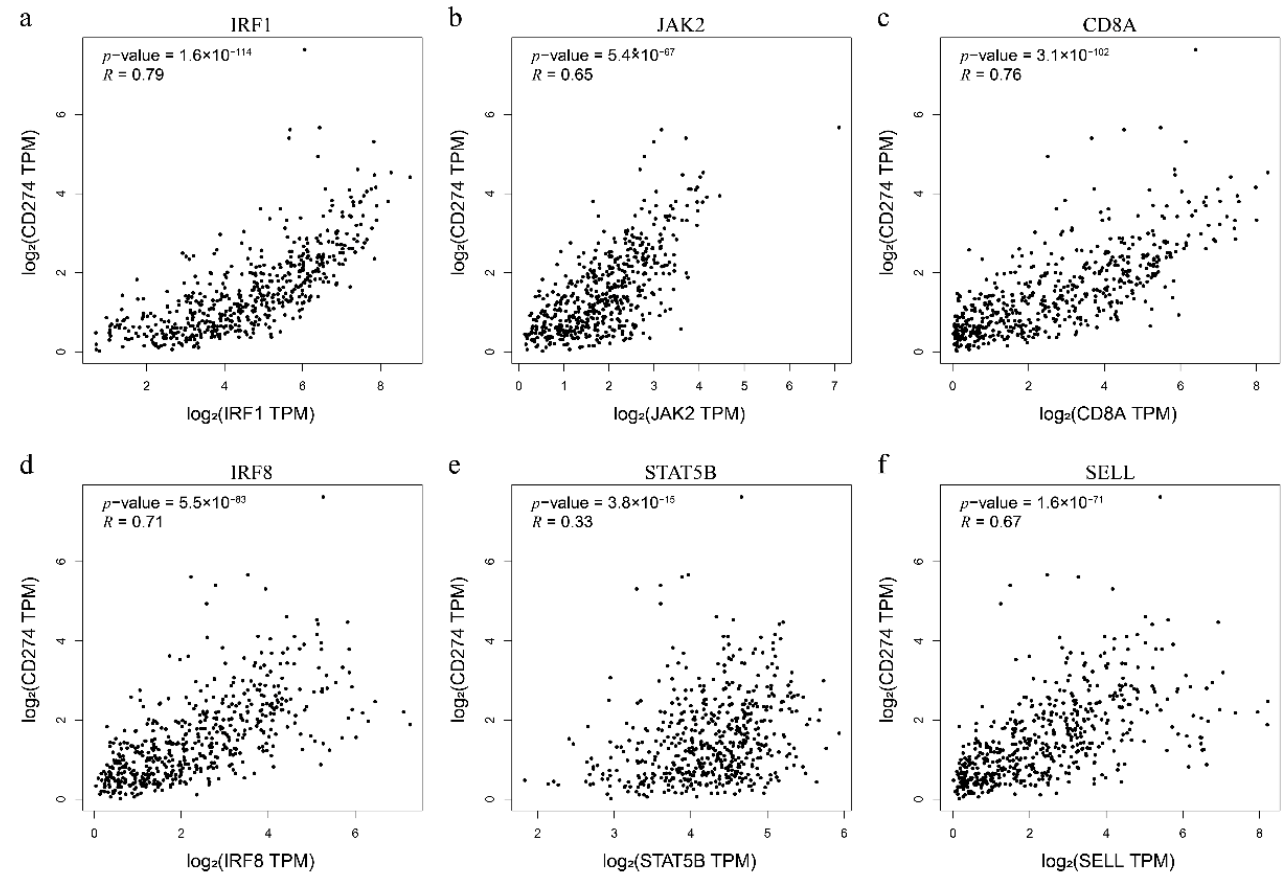

(A)
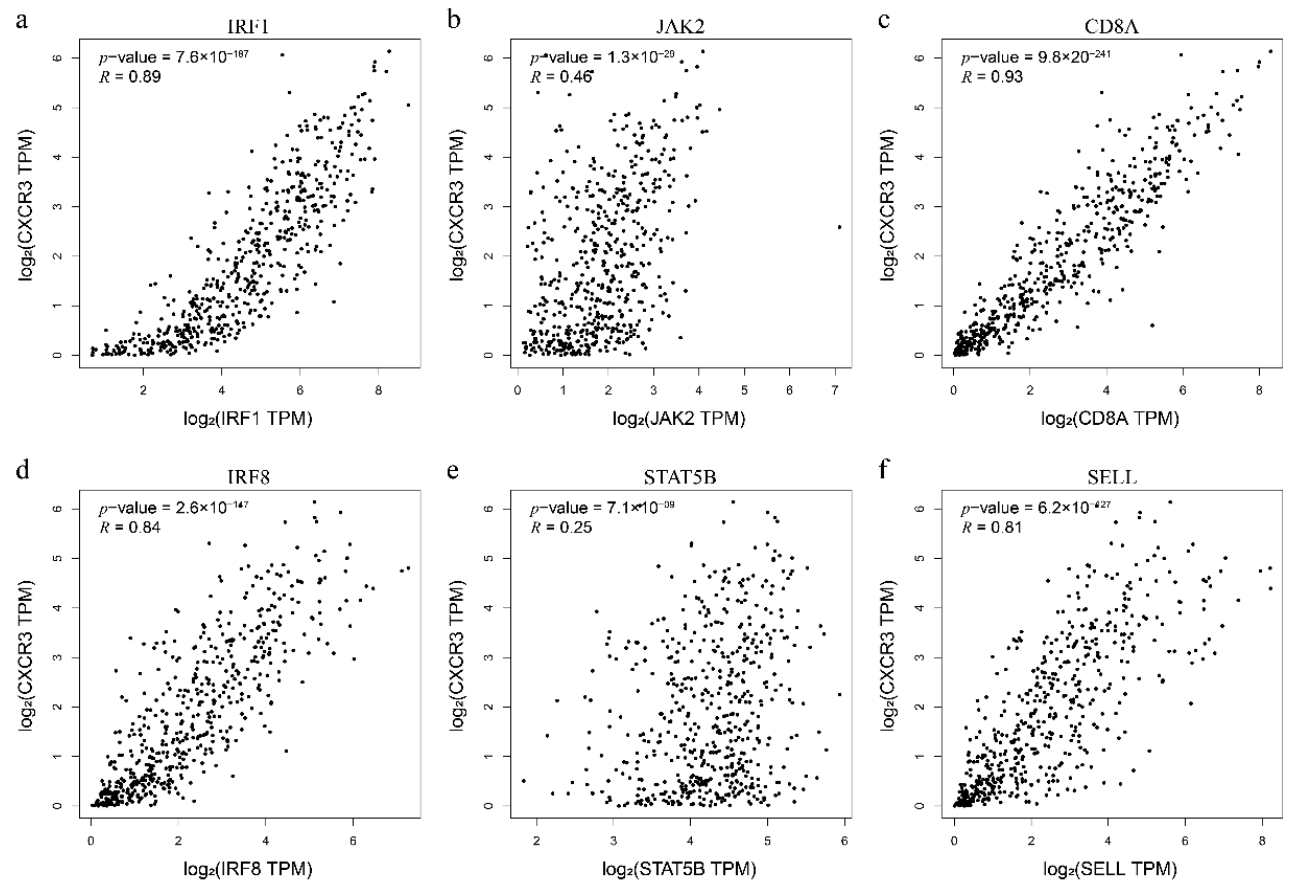

(B)

Figure 9. Cont. 

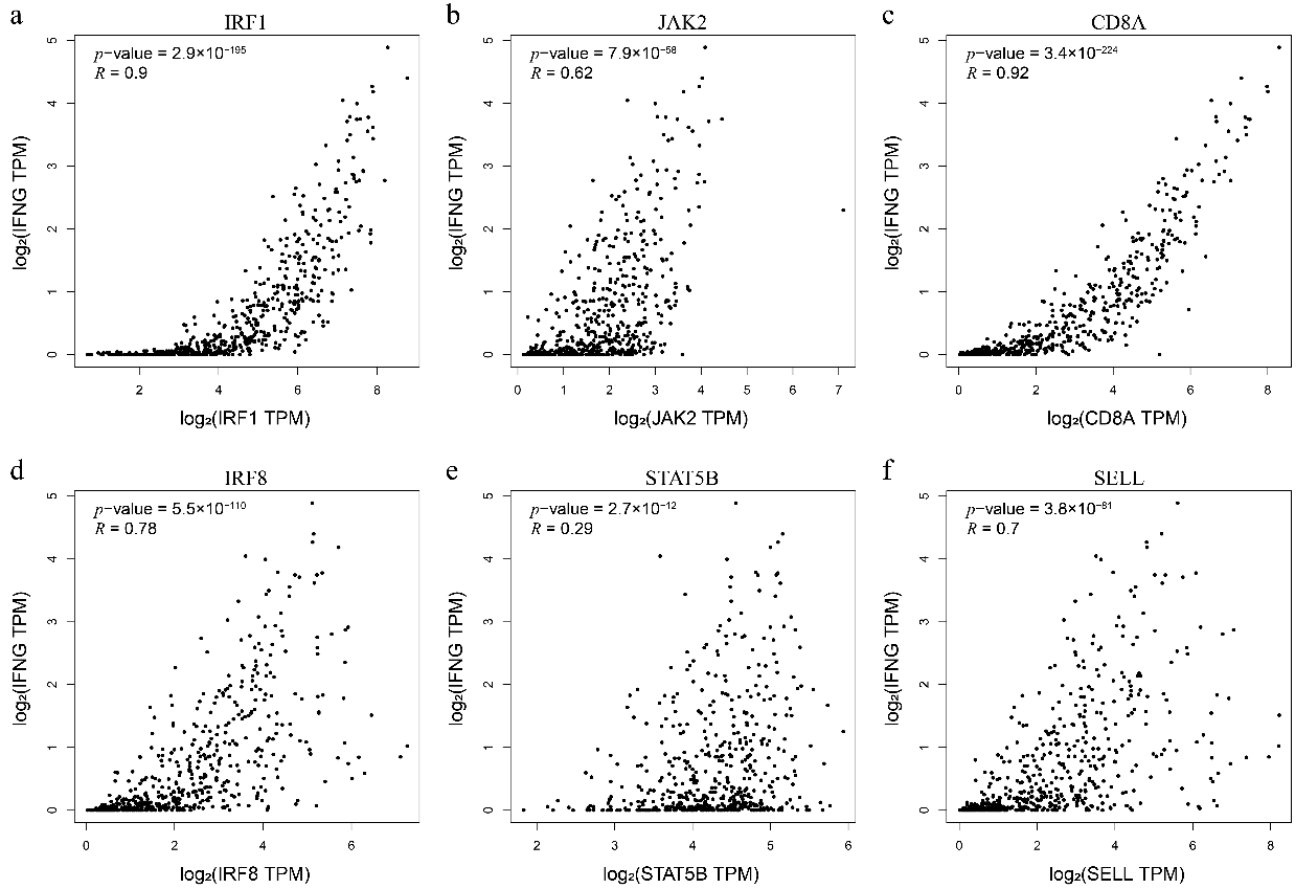

(C)

Figure 9. Expression association analyses between six hub genes and reported biomarkers for anti-PD-1 therapy in melanoma. (A) The relevance of PD-L1 (CD274) and six hub genes. (B) The relevance of CXCR3 and six hub genes. (C) The relevance of IFN- $\gamma$ (IFNG) and six hub genes. Six hub genes including IRF1, JAK2, CD8A, IRF8, STAT5B, and SELL. The x-axis and y-axis represent the expression level $\left(\log _{2}\right.$ TPM) of hub genes and reported melanoma biomarkers of anti-PD-1 therapy, respectively. The upper left corner of the picture shows the $p$-value and correlation coefficient calculated by the Spearman method. TPM: Transcripts per million.

\subsection{The Random Forest Model of Hub Genes}

We constructed a random forest classification model of anti-PD-1 immunotherapy response based on the screened six hub genes (File S1). During the process of building the random forest model, when mtry $=3$, the false positive rate of the model was the lowest (Figure 10A). The optimal model can be achieved when the number of decision trees was about 2,000 (Figure 10B). In addition, the randomForest package provides two indexes to calculate the importance of variables. The one is the index to calculate the prediction error rate based on OOB and is named mean decrease accuracy (\%IncMSE). The other is to calculate the Gini coefficient based on the sample fitting model and is named Mean Decrease Gini (IncNodePurity). The results showed that IRF1 and JAK2 were the more important variables in the prediction model (Figure 10C). Then, the AUC index was used to evaluate the efficiency of the model. The results showed that the prediction model had a good predictive ability for anti-PD-1 immunotherapy response (AUC $=0.75$ ) (Figure 10D). Compared with the single gene, the random forest model had a better value of AUC except for IRF1. Additionally, the samples of an independent dataset GSE93157 $(n=18)$ were used as the validation set to verify the accuracy of the random model. The results also indicated that the random forest model could significantly distinguish the response to anti-PD-1 therapy for melanoma patients (AUC = 0.71) (Figure 10E). 


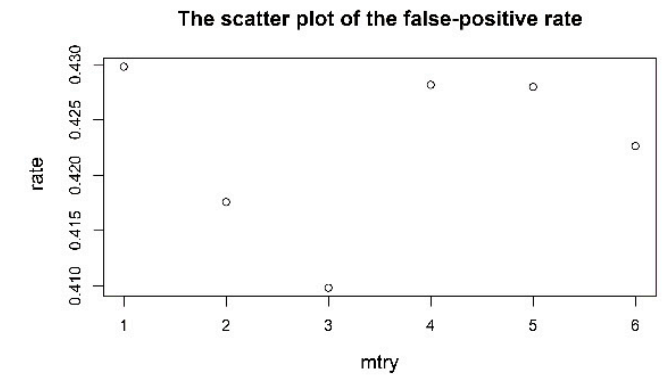

$\mathrm{C}$

The scatter plot of the random forest variable importance measure
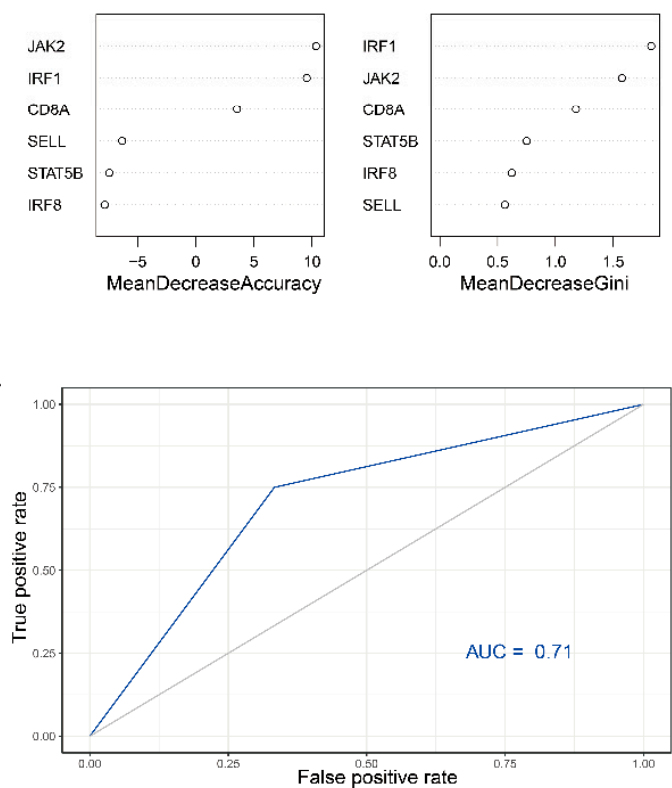

Relationship between the related errors and the number of decision trees
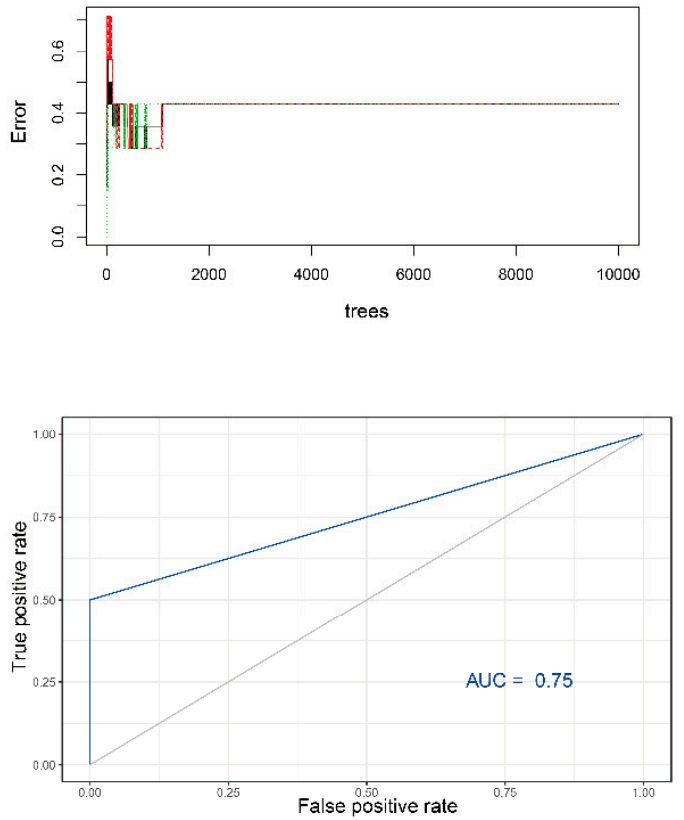

Figure 10. The correlation results of the random forest model. (A) The scatter plot of the false-positive rate. The vertical axis represents the false positive rate, and the horizontal axis represents mtry index. (B) Relationship between the related errors and the number of decision trees in random forests. The vertical axis represents the related errors, and the horizontal axis represents the number of decision trees. (C) The scatter plot of the random forest variable importance measure. The left and right panel were calculated based on the index of mean decrease accuracy and mean decrease Gini, respectively. (D) Evaluation of the prediction efficiency of the random forest model in immunotherapy response (GSE78220). (E) The validation set (GSE93157) verified the accuracy of the random forest model in immunotherapy response.

\section{Discussion}

Despite the significant survival benefits of applying PD-1 checkpoint blockades in patients with melanoma, the response is only observed in a small group, and a massive economic burden comes to the individual patient [51]. Currently, the PD-L1 expression upon IHC is the unique biomarker approved in clinical practice, while its role is still controversial [61]. Therefore, the establishment of predictive biomarkers for immunotherapy response has become a priority.

To investigate potential biomarkers for anti-PD-1 immune checkpoint blockade therapy in melanoma, omics data and network-based approaches were applied in the study. Utilizing gene expression data, a gene co-expression network was built via the WGCNA algorithm to identify key modules related to the clinical features (therapeutic response) [30]. The core genes of the 
clinically significant modules were supposed to be crucial genes in the occurrence and development of disease [62]. Eventually, the pink module was screened, and 13 hub genes were derived from the module. Furthermore, ROC curve analysis showed that six of 13 hub genes including IRF1, JAK2, CD8A, IRF8, STAT5B, and SELL can specifically and accurately distinguish responders from non-responders to anti-PD-1 therapy, which implied the six genes appeared as potential predictors.

To further explore the biological functions of the six hub genes, functional enrichment analysis, survival analysis, immune infiltration level analysis, correlation analysis, and prediction model analysis were enabled. Our results demonstrated that the six hub genes were enriched in lymphocyte proliferation and differentiation as well as immune system regulation. These enriched biological functions contributed to the positive regulation of the immune system and the promotion of anti-tumor immune response [63]. Moreover, the results of survival analysis indicated that the high expression of six hub genes except STAT5B was connected with improved outcomes in melanoma. The multivariate Cox regression analysis implied that high expression of IRF8 was associated with a high risk of death, and high expressions of IRF1, JAK2, CD8A, STAT5B, and SELL were associated with low risk of death. Patients with excellent prognosis frequently revealed positive reactiveness to anti-PD-1 therapy [54]. Thus, we speculated that the high expressions of IRF1, JAK2, CD8A, and SELL might be related to the favorable response of immunotherapy. Besides, our results showed that the expression level of the six hub genes was relevant to TIICs. As a valued factor of anti-tumor immunity, the TIICs can impact the prognosis of patients under immunotherapy [56]. Finally, four genes (IRF1, CD8A, IRF8, and $S E L L)$ were found that closely related to biomarkers of anti-PD-1 therapy (PD-L1, IFN- $\gamma$ and CXCR3). The strong correlation between genes implied that genes may have similar biological functions or regulatory effects [60]. This suggested that the four genes may have a similar ability to predict the anti-PD-1 immunotherapy response. Besides, the random forest prediction model constructed based on the six hub genes showed significant prediction ability of anti-PD-1 therapy. Together with the above findings, there is a strong support of these six hub genes playing pivotal roles in the prognosis and response of checkpoint blockade immunotherapy.

Notably, previous studies provided a large amount of evidence to support the reliability of the results in this study. Rimm, D.L. et al. found that IRF-1 expression was higher in melanoma patients with partial or complete response to anti-PD-1 therapy based on the clinical experiments [64]. Shin, D.S. et al. proposed that JAK2 loss-of-function mutations lead to resistance to PD-1 blockade therapy due to the lack of reactive PD-L1 expression and response to interferon-gamma [65]. Moreover, IRF1 and $J A K 2$ had been reported as biomarkers of the anti-PD-1 immunotherapy response in melanoma via clinical sample validation $[64,66]$. The $C D 8 A$ is a cell surface glycoprotein on most cytotoxic $\mathrm{T}$ lymphocytes, which can mediate efficient cell-cell interactions within the immune system [67]. Wherry, E.J. et al. demonstrated an association between $C D 8 A$ expression in tumors and response to immune checkpoint inhibitors in melanoma [68]. Meanwhile, $C D 8 A$ was conformed as a biomarker to predict the clinical effects of nivolumab in lung cancer [69]. The remaining three hub genes were considered to be closely related to immunotherapy or the immune response. The expression of IRF 8 can selectively induce and maintain the production of soluble factors to regulate the immune response [70]. IRF8 contributed to antitumor immunity due to promoting the differentiation of $\mathrm{CD}^{+}$cells and CD8 ${ }^{+}$cells as well as activation of natural killer cells [71]. STAT5 belongs to the T-cell transcription factor family, which contains two highly related proteins, STAT5A and STAT5B [72]. Auphan-Anezin, N. et al. found targeting STAT5 in tumor-associated immune cells increased the clinical benefits of immunotherapy [73]. Finotto, S. et al. demonstrated that the function of STAT5 can help to improve the effect of lung cancer immunotherapy via detecting the gene expression of STAT5 in CD4 ${ }^{+}$T lymphocytes [72]. Majri, S.S. et al. described STAT5B was a crucial regulator of restimulation-induced T cell death in humans and mice [74]. SELL is a cell adhesion molecule on leukocytes and the preimplantation embryo, which plays crucial roles in lymphocyte-endothelial cell interactions [75]. It was confirmed that SELL can improve the efficacy of cancer immunotherapy via enhancing the activity of T cells [76]. 
Although the expression of $S T A T 5 B$ showed no significant changes between the responders and non-responders to anti-PD-1 therapy based on GSE91061, the gene should not be ignored. STAT5B was the hub gene identified by WGCNA, which may play an important role in the gene network of immunotherapy. ROC analysis indicated that it had significant diagnostic value in the anti-PD-1 therapy response $(\mathrm{AUC}=0.72$, pAUC $=0.80$ ). STAT5B can combine with the other five screened hub genes to construct a random forest prediction model of immunotherapy response with excellent diagnostic efficiency. The results of functional enrichment analysis, immune infection level analysis, correlation analysis and literature validation demonstrated STAT5B was closely associated with the evaluation of response to anti-PD-1 therapy. Therefore, STAT5B was worthy to regard as a potential therapeutic biomarker.

Based on the random forest tool, a prediction model of six genes was constructed to detect the diagnostic efficacy of anti-PD-1 immunotherapy. The results showed that the random forest model of the six genes can distinguish patients that have a response to immunotherapy. However, it is worth noting that not too many samples were used to train the prediction model at present. If the model was directly applied to other datasets, the prediction efficiency may not be satisfactory enough. Therefore, in the future, we will collect more datasets associated with anti-PD-1 therapy in melanoma and continue to retrain our prediction model so that it can be directly extended to other studies. In addition, other limitations of the study were that the six hub genes identified by WGCNA lacked experimental verification. In particular, the results in the present study need to be verified by clinical trials.

In conclusion, six hub genes (IRF1, JAK2, CD8A, IRF8, STAT5B, and SELL) were discovered to distinguish the response of melanoma patients under anti-PD-1 immunotherapy via WGCNA and integrated bioinformatics. These genes may serve as potential biomarkers to guide immunotherapy in the future.

Supplementary Materials: The following are available online at http://www.mdpi.com/2073-4425/11/4/435/s1, Table S1: The top 50 node genes in 11 algorithms, File S1: Construction of random forest model.

Author Contributions: Conceptualization, X.W. and B.L.; methodology, X.W. and Z.C.; formal analysis, F.L. and Z.C.; investigation, X.W. and Z.C.; resources, F.L.; data curation, G.P.; writing-original draft preparation, X.W. and Z.C.; writing-review \& editing, M.L., B.L. and Y.H.; visualization, Z.C.; supervision, M.L.; project administration, X.W.; funding acquisition, M.L. and Y.L. All authors have read and agreed to the published version of the manuscript.

Funding: This work was funded by the Science Innovation Program of College of Laboratory Medicine, Chongqing Medical University (CX201704), Natural Science Foundation of Chongqing, China (No. CSTC2019JCYJ-MSXMX0271, CSTC2019JCYJ-MSXMX0527) and the Science and Technology Research Program of Chongqing Municipal Education Commission (Grant No. KJQN201800523).

Acknowledgments: The authors thank Lu Xie, Shanghai Center for Bioinformation Technology, for helpful suggestions.

Conflicts of Interest: The authors declare no conflicts of interest. The funders had no role in the design of the study; in the collection, analyses, or interpretation of data; in the writing of the manuscript, or in the decision to publish the results.

\section{References}

1. Schadendorf, D.; van Akkooi, A.C.J.; Berking, C.; Griewank, K.G.; Gutzmer, R.; Hauschild, A.; Stang, A.; Roesch, A.; Ugurel, S. Melanoma. Lancet 2018, 392, 971-984. [CrossRef]

2. Gibney, G.T.; Weiner, L.M.; Atkins, M.B. Predictive biomarkers for checkpoint inhibitor-based immunotherapy. Lancet Oncol. 2016, 17, e542-e551. [CrossRef]

3. Topalian, S.L.; Sznol, M.; McDermott, D.F.; Kluger, H.M.; Carvajal, R.D.; Sharfman, W.H.; Brahmer, J.R.; Lawrence, D.P.; Atkins, M.B.; Powderly, J.D.; et al. Survival, durable tumor remission, and long-term safety in patients with advanced melanoma receiving nivolumab. J. Clin. Oncol. 2014, 32, 1020-1030. [CrossRef] [PubMed]

4. Maleki Vareki, S.; Garrigos, C.; Duran, I. Biomarkers of response to PD-1/PD-L1 inhibition. Crit. Rev. Oncol. Hematol. 2017, 116, 116-124. [CrossRef] 
5. Marrone, K.A.; Ying, W.; Naidoo, J. Immune-related adverse events from immune checkpoint inhibitors. Clin. Pharmacol. Ther. 2016, 100, 242-251. [CrossRef]

6. Andrews, A. Treating with checkpoint inhibitors-figure $\$ 1$ million per patient. Am. Health Drug Benefits 2015, 8,9 .

7. Meng, X.; Huang, Z.; Teng, F.; Xing, L.; Yu, J. Predictive biomarkers in PD-1/PD-L1 checkpoint blockade immunotherapy. Cancer Treat. Rev. 2015, 41, 868-876. [CrossRef]

8. Yi, M.; Jiao, D.; Xu, H.; Liu, Q.; Zhao, W.; Han, X.; Wu, K. Biomarkers for predicting efficacy of PD-1/PD-L1 inhibitors. Mol. Cancer 2018, 17, 129. [CrossRef]

9. Teng, F.; Meng, X.; Kong, L.; Yu, J. Progress and challenges of predictive biomarkers of anti PD-1/PD-L1 immunotherapy: A systematic review. Cancer Lett. 2018, 414, 166-173. [CrossRef]

10. Sunshine, J.C.; Nguyen, P.L.; Kaunitz, G.J.; Cottrell, T.R.; Berry, S.; Esandrio, J.; Xu, H.; Ogurtsova, A.; Bleich, K.B.; Cornish, T.C.; et al. PD-L1 expression in melanoma: A quantitative immunohistochemical antibody comparison. Clin. Cancer Res. 2017, 23, 4938-4944. [CrossRef]

11. Cottrell, T.R.; Taube, J.M. PD-L1 and emerging biomarkers in immune checkpoint blockade therapy. Cancer J. 2018, 24, 41-46. [CrossRef]

12. Patel, S.; Osada, T.; Osada, K.; Hurwitz, H.; Lyerly, H.; Morse, M. Modulation of immune system inhibitory checkpoints in colorectal cancer. Curr. Colorectal Cancer Rep. 2013, 9, 391-397. [CrossRef]

13. Johnson, D.B.; Bordeaux, J.; Kim, J.Y.; Vaupel, C.; Rimm, D.L.; Ho, T.H.; Joseph, R.W.; Daud, A.I.; Conry, R.M.; Gaughan, E.M.; et al. Quantitative spatial profiling of PD-1/PD-L1 interaction and HLA-DR/IDO-1 predicts improved outcomes of anti-PD-1 therapies in metastatic melanoma. Clin. Cancer Res. 2018, 24, 5250-5260. [CrossRef] [PubMed]

14. Vilain, R.E.; Menzies, A.M.; Wilmott, J.S.; Kakavand, H.; Madore, J.; Guminski, A.; Liniker, E.; Kong, B.Y.; Cooper, A.J.; Howle, J.R.; et al. Dynamic changes in PD-L1 expression and immune infiltrates early during treatment predict response to PD-1 blockade in melanoma. Clin. Cancer Res. 2017, 23, 5024-5033. [CrossRef] [PubMed]

15. Yue, C.; Jiang, Y.; Li, P.; Wang, Y.; Xue, J.; Li, N.; Li, D.; Wang, R.; Dang, Y.; Hu, Z.; et al. Dynamic change of PD-L1 expression on circulating tumor cells in advanced solid tumor patients undergoing PD-1 blockade therapy. Oncoimmunology 2018, 7, e1438111. [CrossRef]

16. Danilova, L.; Wang, H.; Sunshine, J.; Kaunitz, G.J.; Cottrell, T.R.; Xu, H.; Esandrio, J.; Anders, R.A.; Cope, L.; Pardoll, D.M.; et al. Association of PD-1/PD-L axis expression with cytolytic activity, mutational load, and prognosis in melanoma and other solid tumors. Proc. Natl. Acad. Sci. USA 2016, 113, E7769-E7777. [CrossRef]

17. Messina, J.L.; Fenstermacher, D.A.; Eschrich, S.; Qu, X.; Berglund, A.E.; Lloyd, M.C.; Schell, M.J.; Sondak, V.K.; Weber, J.S.; Mule, J.J. 12-chemokine gene signature identifies lymph node-like structures in melanoma: Potential for patient selection for immunotherapy? Sci. Rep. 2012, 2, 765. [CrossRef]

18. Ayers, M.; Lunceford, J.; Nebozhyn, M.; Murphy, E.; Loboda, A.; Kaufman, D.R.; Albright, A.; Cheng, J.D.; Kang, S.P.; Shankaran, V.; et al. IFN- $\gamma$-related mrna profile predicts clinical response to PD-1 blockade. J. Clin. Investig. 2017, 127, 2930-2940. [CrossRef]

19. Ribas, A.; Robert, C.; Hodi, F.S.; Wolchok, J.D.; Joshua, A.; Hwu, W.-J. Association of response to programmed death receptor 1 (PD-1) blockade with pembrolizumab (MK-3475) with an interferon-inflammatory immune gene signature. J. Clin. Oncol. 2015, 33, 3001. [CrossRef]

20. Varn, F.S.; Wang, Y.; Cheng, C. A B cell-derived gene expression signature associates with an immunologically active tumor microenvironment and response to immune checkpoint blockade therapy. Oncoimmunology 2019, 8, e1513440. [CrossRef]

21. Zuo, Z.; Shen, J.X.; Pan, Y.; Pu, J.; Li, Y.G.; Shao, X.H.; Wang, W.P. Weighted gene correlation network analysis (WGCNA) detected loss of MAGI2 promotes chronic kidney disease (CKD) by podocyte damage. Cell Physiol. Biochem. 2018, 51, 244-261. [CrossRef] [PubMed]

22. Wang, W.; Fu, S.; Lin, X.; Zheng, J.; Pu, J.; Gu, Y.; Deng, W.; Liu, Y.; He, Z.; Liang, W.; et al. miR-92b-3p functions as a key gene in esophageal squamous cell cancer as determined by co-expression analysis. Onco Targets Ther. 2019, 12, 8339-8353. [CrossRef] [PubMed]

23. Clough, E.; Barrett, T. The gene expression omnibus database. Methods Mol. Biol 2016, 1418, 93-110. [PubMed] 
24. Parkinson, H.; Kapushesky, M.; Shojatalab, M.; Abeygunawardena, N.; Coulson, R.; Farne, A.; Holloway, E.; Kolesnykov, N.; Lilja, P.; Lukk, M.; et al. Arrayexpress-A public database of microarray experiments and gene expression profiles. Nucleic Acids Res. 2007, 35, D747-D750. [CrossRef] [PubMed]

25. Papatheodorou, I.; Fonseca, N.A.; Keays, M.; Tang, Y.A.; Barrera, E.; Bazant, W.; Burke, M.; Fullgrabe, A.; Fuentes, A.M.; George, N.; et al. Expression atlas: Gene and protein expression across multiple studies and organisms. Nucleic Acids Res. 2018, 46, D246-D251. [CrossRef] [PubMed]

26. Seymour, L.; Bogaerts, J.; Perrone, A.; Ford, R.; Schwartz, L.H.; Mandrekar, S.; Lin, N.U.; Litiere, S.; Dancey, J.; Chen, A.; et al. Irecist: Guidelines for response criteria for use in trials testing immunotherapeutics. Lancet Oncol. 2017, 18, e143-e152. [CrossRef]

27. Riaz, N.; Havel, J.J.; Makarov, V.; Desrichard, A.; Urba, W.J.; Sims, J.S.; Hodi, F.S.; Martin-Algarra, S.; Mandal, R.; Sharfman, W.H.; et al. Tumor and microenvironment evolution during immunotherapy with nivolumab. Cell 2017, 171, 934-949.e916. [CrossRef]

28. Hugo, W.; Zaretsky, J.M.; Sun, L.; Song, C.; Moreno, B.H.; Hu-Lieskovan, S.; Berent-Maoz, B.; Pang, J.; Chmielowski, B.; Cherry, G.; et al. Genomic and transcriptomic features of response to anti-PD-1 therapy in metastatic melanoma. Cell 2016, 165, 35-44. [CrossRef]

29. Anders, S.; Huber, W. Differential expression analysis for sequence count data. Genome Biol 2010, 11, R106. [CrossRef]

30. Langfelder, P.; Horvath, S. WGCNA: An R package for weighted correlation network analysis. BMC Bioinform. 2008, 9, 559. [CrossRef]

31. Zhou, G.; Soufan, O.; Ewald, J.; Hancock, R.E.W.; Basu, N.; Xia, J. Networkanalyst 3.0: A visual analytics platform for comprehensive gene expression profiling and meta-analysis. Nucleic Acids Res. 2019, 47, W234-W241. [CrossRef] [PubMed]

32. Szklarczyk, D.; Gable, A.L.; Lyon, D.; Junge, A.; Wyder, S.; Huerta-Cepas, J.; Simonovic, M.; Doncheva, N.T.; Morris, J.H.; Bork, P.; et al. STRING v11: Protein-protein association networks with increased coverage, supporting functional discovery in genome-wide experimental datasets. Nucleic Acids Res. 2019, 47, D607-D613. [CrossRef] [PubMed]

33. Chin, C.H.; Chen, S.H.; Wu, H.H.; Ho, C.W.; Ko, M.T.; Lin, C.Y. Cytohubba: Identifying hub objects and sub-networks from complex interactome. BMC Syst. Biol. 2014, 8, S11. [CrossRef] [PubMed]

34. Shannon, P.; Markiel, A.; Ozier, O.; Baliga, N.S.; Wang, J.T.; Ramage, D.; Amin, N.; Schwikowski, B.; Ideker, T. Cytoscape: A software environment for integrated models of biomolecular interaction networks. Genome Res. 2003, 13, 2498-2504. [CrossRef] [PubMed]

35. Robin, X.; Turck, N.; Hainard, A.; Tiberti, N.; Lisacek, F.; Sanchez, J.C.; Muller, M. Proc: An open-source package for R and S+ to analyze and compare ROC curves. BMC Bioinformatics 2011, 12, 77. [CrossRef]

36. Hsu, M.J.; Chang, Y.C.; Hsueh, H.M. Biomarker selection for medical diagnosis using the partial area under the ROC curve. BMC Res. Notes 2014, 7, 25. [CrossRef]

37. Hajian-Tilaki, K. Receiver operating characteristic (ROC) curve analysis for medical diagnostic test evaluation. Caspian J. Intern. Med. 2013, 4, 627-635.

38. Hanzelmann, S.; Castelo, R.; Guinney, J. GSVA: Gene set variation analysis for microarray and RNA-seq data. BMC Bioinformatics 2013, 14, 7. [CrossRef]

39. Li, T.; Fan, J.; Wang, B.; Traugh, N.; Chen, Q.; Liu, J.S.; Li, B.; Liu, X.S. Timer: A web server for comprehensive analysis of tumor-infiltrating immune cells. Cancer Res. 2017, 77, e108-e110. [CrossRef]

40. Li, B.; Severson, E.; Pignon, J.C.; Zhao, H.; Li, T.; Novak, J.; Jiang, P.; Shen, H.; Aster, J.C.; Rodig, S.; et al. Comprehensive analyses of tumor immunity: Implications for cancer immunotherapy. Genome Biol. 2016, 17, 174. [CrossRef]

41. Tang, Z.; Li, C.; Kang, B.; Gao, G.; Li, C.; Zhang, Z. GEPIA: A web server for cancer and normal gene expression profiling and interactive analyses. Nucleic Acids Res. 2017, 45, W98-W102. [CrossRef] [PubMed]

42. Sitbon, O.; McLaughlin, V.V.; Badesch, D.B.; Barst, R.J.; Black, C.; Galie, N.; Humbert, M.; Rainisio, M.; Rubin, L.J.; Simonneau, G. Survival in patients with class III idiopathic pulmonary arterial hypertension treated with first line oral bosentan compared with an historical cohort of patients started on intravenous epoprostenol. Thorax 2005, 60, 1025-1030. [CrossRef] [PubMed]

43. Breiman, L. Random forests. Machine Learning 2001, 45, 5-32. [CrossRef]

44. Genuer, R.; Poggi, J.; Tuleau-Malot, C. Variable selection using random forests. Pattern Recogn. Lett. 2010, 31, 2225-2236. [CrossRef] 
45. Prat, A.; Navarro, A.; Pare, L.; Reguart, N.; Galvan, P.; Pascual, T.; Martinez, A.; Nuciforo, P.; Comerma, L.; Alos, L.; et al. Immune-related gene expression profiling after PD-1 blockade in non-small cell lung carcinoma, head and neck squamous cell carcinoma, and melanoma. Cancer Res. 2017, 77, 3540-3550. [CrossRef]

46. Chen, D.S.; Mellman, I. Elements of cancer immunity and the cancer-immune set point. Nature 2017, 541, 321-330. [CrossRef]

47. Hsu, J.; Hodgins, J.J.; Marathe, M.; Nicolai, C.J.; Bourgeois-Daigneault, M.C.; Trevino, T.N.; Azimi, C.S.; Scheer, A.K.; Randolph, H.E.; Thompson, T.W.; et al. Contribution of NK cells to immunotherapy mediated by PD-1/PD-L1 blockade. J. Clin. Investig. 2018, 128, 4654-4668. [CrossRef]

48. Herbst, R.S.; Soria, J.C.; Kowanetz, M.; Fine, G.D.; Hamid, O.; Gordon, M.S.; Sosman, J.A.; McDermott, D.F.; Powderly, J.D.; Gettinger, S.N.; et al. Predictive correlates of response to the anti-PD-L1 antibody MPDL3280A in cancer patients. Nature 2014, 515, 563-567. [CrossRef]

49. Dulos, J.; Carven, G.J.; van Boxtel, S.J.; Evers, S.; Driessen-Engels, L.J.; Hobo, W.; Gorecka, M.A.; de Haan, A.F.; Mulders, P.; Punt, C.J.; et al. PD-1 blockade augments Th1 and Th17 and suppresses Th2 responses in peripheral blood from patients with prostate and advanced melanoma cancer. J. Immunother. 2012, 35, 169-178. [CrossRef]

50. Lu, C.; Talukder, A.; Savage, N.M.; Singh, N.; Liu, K. JAK-STAT-mediated chronic inflammation impairs cytotoxic T lymphocyte activation to decrease anti-PD-1 immunotherapy efficacy in pancreatic cancer. Oncoimmunology 2017, 6, e1291106. [CrossRef]

51. Havel, J.J.; Chowell, D.; Chan, T.A. The evolving landscape of biomarkers for checkpoint inhibitor immunotherapy. Nat. Rev. Cancer 2019, 19, 133-150. [CrossRef] [PubMed]

52. Garris, C.S.; Arlauckas, S.P.; Kohler, R.H.; Trefny, M.P.; Garren, S.; Piot, C.; Engblom, C.; Pfirschke, C.; Siwicki, M.; Gungabeesoon, J.; et al. Successful anti-PD-1 cancer immunotherapy requires T cell-dendritic cell crosstalk involving the cytokines IFN- $\gamma$ and IL-12. Immunity 2018, 49, 1148-1161.e1147. [CrossRef] [PubMed]

53. Saravia, D.; Okabe, N.; Park, W.; Kwon, D.; Mezquita, L.; Chae, Y.; Mudad, R.; Jahanzeb, M.; Besse, B.; Suzuki, H.; et al. Neutrophil-lymphocyte-ratio to complement the prediction ability of PD-L1 expression for outcomes in patients with advanced non-small cell lung cancer treated with PD-1/ PD-L1 inhibitors. J. Clin. Oncol. 2018, 36, e15102. [CrossRef]

54. Gauci, M.L.; Lanoy, E.; Champiat, S.; Caramella, C.; Ammari, S.; Aspeslagh, S.; Varga, A.; Baldini, C.; Bahleda, R.; Gazzah, A.; et al. Long-term survival in patients responding to anti- PD-1/ PD-L1 therapy and disease outcome upon treatment discontinuation. Clin. Cancer Res. 2019, 25, 946-956. [CrossRef] [PubMed]

55. Pitt, J.M.; Vetizou, M.; Daillere, R.; Roberti, M.P.; Yamazaki, T.; Routy, B.; Lepage, P.; Boneca, I.G.; Chamaillard, M.; Kroemer, G.; et al. Resistance mechanisms to immune-checkpoint blockade in cancer: Tumor-intrinsic and -extrinsic factors. Immunity 2016, 44, 1255-1269. [CrossRef] [PubMed]

56. Nishino, M.; Ramaiya, N.H.; Hatabu, H.; Hodi, F.S. Monitoring immune-checkpoint blockade: Response evaluation and biomarker development. Nat. Rev. Clin. Oncol. 2017, 14, 655-668. [CrossRef]

57. Liu, C.; He, H.; Li, X.; Su, M.A.; Cao, Y. Dynamic metrics-based biomarkers to predict responders to anti-PD-1 immunotherapy. Br. J. Cancer 2019, 120, 346-355. [CrossRef]

58. Han, X.; Wang, Y.; Sun, J.; Tan, T.; Cai, X.; Lin, P.; Tan, Y.; Zheng, B.; Wang, B.; Wang, J.; et al. Role of CXCR3 signaling in response to anti-PD-1 therapy. EBioMedicine 2019, 48, 169-177. [CrossRef]

59. Chow, M.T.; Ozga, A.J.; Servis, R.L.; Frederick, D.T.; Lo, J.A.; Fisher, D.E.; Freeman, G.J.; Boland, G.M.; Luster, A.D. Intratumoral activity of the CXCR3 chemokine system is required for the efficacy of anti-PD-1 therapy. Immunity 2019, 50, 1498-1512.e1495. [CrossRef]

60. Reynier, F.; Petit, F.; Paye, M.; Turrel-Davin, F.; Imbert, P.E.; Hot, A.; Mougin, B.; Miossec, P. Importance of correlation between gene expression levels: Application to the type I interferon signature in rheumatoid arthritis. PLoS ONE 2011, 6, e24828. [CrossRef]

61. Patel, S.P.; Kurzrock, R. PD-L1 expression as a predictive biomarker in cancer immunotherapy. Mol. Cancer Ther. 2015, 14, 847-856. [CrossRef] [PubMed]

62. Giulietti, M.; Occhipinti, G.; Principato, G.; Piva, F. Identification of candidate mirna biomarkers for pancreatic ductal adenocarcinoma by weighted gene co-expression network analysis. Cell Oncol. (Dordr.) 2017, 40, 181-192. [CrossRef] [PubMed]

63. Heinzel, S.; Marchingo, J.M.; Horton, M.B.; Hodgkin, P.D. The regulation of lymphocyte activation and proliferation. Curr. Opin. Immunol. 2018, 51, 32-38. [CrossRef] [PubMed] 
64. Smithy, J.W.; Moore, L.M.; Pelekanou, V.; Rehman, J.; Gaule, P.; Wong, P.F.; Neumeister, V.M.; Sznol, M.; Kluger, H.M.; Rimm, D.L. Nuclear IRF-1 expression as a mechanism to assess "capability" to express PD-L1 and response to PD-1 therapy in metastatic melanoma. J. Immunother. Cancer 2017, 5, 25. [CrossRef] [PubMed]

65. Shin, D.S.; Zaretsky, J.M.; Escuin-Ordinas, H.; Garcia-Diaz, A.; Hu-Lieskovan, S.; Kalbasi, A.; Grasso, C.S.; Hugo, W.; Sandoval, S.; Torrejon, D.Y; et al. Primary resistance to PD-1 blockade mediated by JAK1/2 mutations. Cancer Discov. 2017, 7, 188-201. [CrossRef]

66. Peng, J.; Xiao, L.-S.; Dong, Z.-Y.; Li, W.-W.; Wang, K.-Y.; Wu, D.-H.; Liu, L. Potential predictive value of JAK2 expression for pan-cancer response to PD-1 blockade immunotherapy. Transl. Cancer Res. 2018, 7, 462-471. [CrossRef]

67. Kioussis, D.; Ellmeier, W. Chromatin and CD4, CD8A and CD8B gene expression during thymic differentiation. Nat. Rev. Immunol. 2002, 2, 909-919. [CrossRef]

68. Huang, A.C.; Postow, M.A.; Orlowski, R.J.; Mick, R.; Bengsch, B.; Manne, S.; Xu, W.; Harmon, S.; Giles, J.R.; Wenz, B.; et al. T-cell invigoration to tumour burden ratio associated with anti-PD-1 response. Nature 2017, 545, 60-65. [CrossRef]

69. Fumet, J.D.; Richard, C.; Ledys, F.; Klopfenstein, Q.; Joubert, P.; Routy, B.; Truntzer, C.; Gagne, A.; Hamel, M.A.; Guimaraes, C.F.; et al. Prognostic and predictive role of CD8 and PD-L1 determination in lung tumor tissue of patients under anti-PD-1 therapy. Br. J. Cancer 2018, 119, 950-960. [CrossRef]

70. Schiavoni, G.; Gabriele, L.; Mattei, F. The dual role of IRF8 in cancer immunosurveillance. Oncoimmunology 2013, 2, e25476. [CrossRef]

71. Muhitch, J.B.; Hoffend, N.C.; Azabdaftari, G.; Miller, A.; Bshara, W.; Morrison, C.D.; Schwaab, T.; Abrams, S.I. Tumor-associated macrophage expression of interferon regulatory factor-8 (IRF8) is a predictor of progression and patient survival in renal cell carcinoma. J. Immunother. Cancer 2019, 7, 155. [CrossRef] [PubMed]

72. Friedrich, J.M.M.M.; Heim, L.; Springel, R.; Mittler, S.; Gafencu, D.; Hartmann, A.; Sirbu, H.; Finotto, S. The role of signal transducer and activator of transcription protein 5 (STAT5) in non-small cell lung cancer (NSCLC). J. Immunol. 2016, 196, 73.18.

73. Verdeil, G.; Lawrence, T.; Schmitt-Verhulst, A.M.; Auphan-Anezin, N. Targeting STAT3 and STAT5 in tumor-associated immune cells to improve immunotherapy. Cancers (Basel) 2019, 11, 1832. [CrossRef]

74. Majri, S.S.; Fritz, J.M.; Villarino, A.V.; Zheng, L.; Kanellopoulou, C.; Chaigne-Delalande, B.; Gronholm, J.; Niemela, J.E.; Afzali, B.; Biancalana, M.; et al. STAT5B: A differential regulator of the life and death of CD4(+) effector memory T cells. J. Immunol. 2018, 200, 110-118. [CrossRef] [PubMed]

75. Ivetic, A.; Hoskins Green, H.L.; Hart, S.J. L-selectin: A major regulator of leukocyte adhesion, migration and signaling. Front. Immunol. 2019, 10, 1068. [CrossRef]

76. Watson, H.A.; Durairaj, R.R.P.; Ohme, J.; Alatsatianos, M.; Almutairi, H.; Mohammed, R.N.; Vigar, M.; Reed, S.G.; Paisey, S.J.; Marshall, C.; et al. L-selectin enhanced T cells improve the efficacy of cancer immunotherapy. Front. Immunol. 2019, 10, 1321. [CrossRef] 